\title{
FOLDING MODEL STUDY OF THE ELASTIC ALPHA-ALPHA SCATTERING AT LOW ENERGIES
}

\author{
NGO HAI TAN, NGUYEN HOANG PHUC, AND DAO TIEN KHOA \\ Institute for Nuclear Science \& Technology, Vinatom \\ 179 Hoang Quoc Viet Road, Cau Giay, Hanoi \\ Received 04 November 2013 \\ Accepted for publication 31 December 2013
}

\begin{abstract}
The folding model analysis of the elastic $\alpha+\alpha$ scattering at the incident energies below the reaction threshold of $34.7 \mathrm{MeV}$ (in the lab system) has been done using the well-tested density dependent versions of the M3Y interaction and realistic choices for the ${ }^{4} H e$ density. Because the absorption is negligible at the energies below the reaction threshold, we were able to probe the $\alpha+\alpha$ optical potential at low energies quite unambiguously and found that the $\alpha+\alpha$ overlap density used to construct the density dependence of the M3Y interaction is strongly distorted by the Pauli blocking. This result gives possible explanation of a long-standing inconsistency of the double-folding model in its study of the elastic $\alpha+\alpha$ and $\alpha$-nucleus scattering at low energies using the same realistic density dependent M3Y interaction.
\end{abstract}

\section{INTRODUCTION}

The knowledge about the $\alpha+\alpha$ interaction at low energies is of fundamental importance due, in part, to diversities of the $\alpha$-cluster phenomena in nuclear physics, where one has to deal with configurations of two or more $\alpha$-particles interacting with each other. Thanks to the robust, tightly bound structure of the (spin- and isospin zero) ${ }^{4} \mathrm{He}$ nucleus, the elastic $\alpha+\alpha$ scattering cross section has been measured quite accurately during the sixties and seventies of the last century, and numerous phase-shift analyses were made based on these cross sections $[1,2]$. The key quantity in an optical model (OM) study of elastic $\alpha+\alpha$ scattering is the $\alpha+\alpha$ optical potential (OP) that has been treated either phenomenologically [1] or evaluated microscopically from the (two-body) nucleon-nucleon $(\mathrm{NN})$ interaction between nucleons bound in the two interacting $\alpha$-particles [3, 4]. In terms of the quantum mechanical treatment of elastic $\alpha+\alpha$ scattering, the resonating group method (RGM) (see, e.g., Refs. $[3,5]$ ) is the most rigorous approach that takes into account the full antisymmetrization of the total wave function of the scattering system. The one-body wave equation for the relative wave function $\chi(R)$ is then constructed with a nonlocal RGM potential kernel. An accurate localization approximation has also been developed [5] to yield a local optical potential $U(R)$ to be used in the standard OM equation to determine $\chi(R)$. Given a complicated treatment of the nonlocal exchange kernel, only a density independent NN interaction in the Gaussian form could be used as the effective interaction in the RGM calculation. The Pauli blocking effects have also been 
studied rigorously in the fish-bone model for the $\alpha+\alpha$ potential [6]. In a somewhat less rigorous way, the double-folding model (see, e.g., Refs. [4,7-10]) determines the OP for the $\alpha+\alpha$ system as

$$
U=\sum_{i \in \alpha_{1} ; j \in \alpha_{2}}\left[\left\langle i j\left|v_{\mathrm{D}}\right| i j\right\rangle+\left\langle i j\left|v_{\mathrm{EX}}\right| j i\right\rangle\right]
$$

where $v_{\mathrm{D}(\mathrm{EX})}$ are the direct and exchange parts of the effective NN interaction between nucleons in the first $\alpha$-particle and those in the second one. The antisymmetrization gives rise to the exchange term in Eq. (1) that is, in general, nonlocal in the coordinate space. To have a local double-folded OP, an accurate local approximation for the exchange potential has been developed [7-9], which allowed the use of some realistic density dependent NN interaction. Among different choices of the effective NN interaction, the original density independent M3Y interactions [11,12] have been used with some success in the doublefolding calculations of the heavy-ion (HI) optical potential at low energies [4], where the data are sensitive only to the potential at the surface because of the strong absorption. However, in cases of refractive (rainbow) nucleus-nucleus scattering where the elastic data are sensitive to the OP over a much wider radial range, the density independent M3Y interactions failed to give a good description of the data and the inclusion of an explicit density dependence was found necessary [13] to account for the reduction of the attractive strength of the in-medium NN interaction that occurs as the density of the nuclear medium increases. Such an effect has been shown to be due to the saturation properties of nuclear matter and some realistic density dependent versions of the M3Y interaction $[8,10]$ have been introduced and used successfully in the folding model analysis of the elastic $\alpha$-nucleus scattering (see the recent review in Ref. [9]), and it is natural to expect the same success of this density dependent interaction in the study of the elastic $\alpha+\alpha$ scattering. The actual double-folding calculation has shown, however, that only the original density independent M3Y interaction can give a reasonable description of the elastic $\alpha+\alpha$ scattering at low energies [14]. Such an inconsistency of the double-folding model has also been noted earlier in Ref. [4], where the rainbow $\alpha$-nucleus scattering data implied the inclusion of a realistic density dependence into the M3Y interaction, while the elastic $\alpha+\alpha$ scattering data preferred the original density independent M3Y interaction.

In contrast to HI scattering, the elastic $\alpha+\alpha$ scattering data at energies below the reaction threshold of $34.7 \mathrm{MeV}$ (in the lab system) can be well described by the real OP only $[1,3,4,14]$, neglecting the imaginary (absorptive) part of the OP. Without the absorption, the elastic $\alpha+\alpha$ data measured accurately over the whole observable angular range should be sensitive to the real OP down to small radii where the density dependent effects should be substantial due to a high $\alpha+\alpha$ overlap density. Thus, the success of the density independent M3Y interaction in the description of the considered $\alpha+\alpha$ data indicates likely to a strong depletion of the $\alpha+\alpha$ overlap density that suppresses the density dependent effects on the shape and depth of the $\alpha+\alpha$ potential.

To shed more light on the applicability of the double-folding model in the study of the $\alpha+\alpha$ scattering at low energies, we have performed in the present work a detailed folding model analysis of the available elastic $\alpha+\alpha$ data at energies below the reaction threshold. The effects of the density dependence of the NN interaction to the $\alpha+\alpha$ 
potential were studied carefully, based on different assumptions for the $\alpha+\alpha$ overlap density.

\section{THEORETICAL FORMALISM}

Our microscopic study of the elastic $\alpha+\alpha$ scattering is based on the double-folding model (DFM) [7], which calculates the real OP of the $\alpha+\alpha$ system using the ground state density of ${ }^{4} \mathrm{He}$ nucleus and an appropriate choice of the effective NN interaction. $\alpha$-particle is a unique case when a simple Gaussian can reproduce rather well its ground state (g.s) density. Like in numerous folding model studies of $\alpha$-nucleus scattering, we have used in the present DFM calculation the Gaussian form for the $\alpha$-density suggested by Satchler and Love [4]. This $\alpha$-density has a RMS radius of $1.461 \mathrm{fm}$, close to the empirical value of $1.47 \pm 0.02 \mathrm{fm}$ that can be deduced from the experimental charge density of ${ }^{4} \mathrm{He}[16,17]$.

It is straightforward to see that the Gaussian density is readily obtained in a simple 4-nucleon model for the $\alpha$-particle, where 4 nucleons occupy the lowest $\mathrm{s} \frac{1}{2}$ harmonic oscillator (h.o.) shell [15]. After the spurious center-of-mass (c.m.) component is excluded from the 4-nucleon wave function using the prescription of Ref. [18], the $\alpha$-density remains in a Gaussian form but with a modified h.o. range $b$

$$
\rho(r)=\frac{4}{\pi^{3 / 2} b^{3}} \exp \left(-\frac{r^{2}}{b^{2}}\right) \rightarrow \text { c.m. correction } \rightarrow \rho(r)=\frac{32}{(3 \pi)^{3 / 2} b^{3}} \exp \left(-\frac{4 r^{2}}{3 b^{2}}\right) .
$$

Therefore, if one assumes $\sqrt{3} b / 2=1.1932 \mathrm{fm}$ in the $\alpha$-density after the c.m. correction then it turns out to be the same Gaussian as that suggested by Satchler and Love [4], which has been used so far in most of the folding calculations of the $\alpha+\alpha$ and $\alpha$-nucleus potentials $[4,8,9,13-15]$. In the present work we have compared in some cases the results obtained with the Gaussian density (2) with those obtained with the experimental $\alpha$ density (twice the experimental ${ }^{4} \mathrm{He}$ charge density [16] unfolded with the finite size of proton).

\section{II.1. Density dependent M3Y interaction}

A popular choice of the effective NN interaction for the DFM calculation has been one of the M3Y interactions that were designed to reproduce the G-matrix elements of the Reid [11] and Paris [12] NN potentials in an oscillator basis. Although the original density independent M3Y interaction has been used with some success to calculate the real HI optical potential at low energies, where the scattering data are sensitive to the real OP only at the surface [4], it failed to account for the nuclear 'rainbow' scattering (observed first in the elastic $\alpha$-nucleus scattering and later on in some light heavy-ion systems [9]), when the scattering data are sensitive to the real OP over a wider radial range. This has motivated the inclusion of an explicit density dependence into the original M3Y interaction $[8,10,13]$ to properly account for the reduction of the attractive strength of the effective NN interaction occurring at high densities of the nuclear medium (see Fig. 1).

We have chosen for the present study the BDM3Y1 [10] and CDM3Y6 [8] density dependent versions of the M3Y interaction that are based on the original M3Y-Reid [11] 


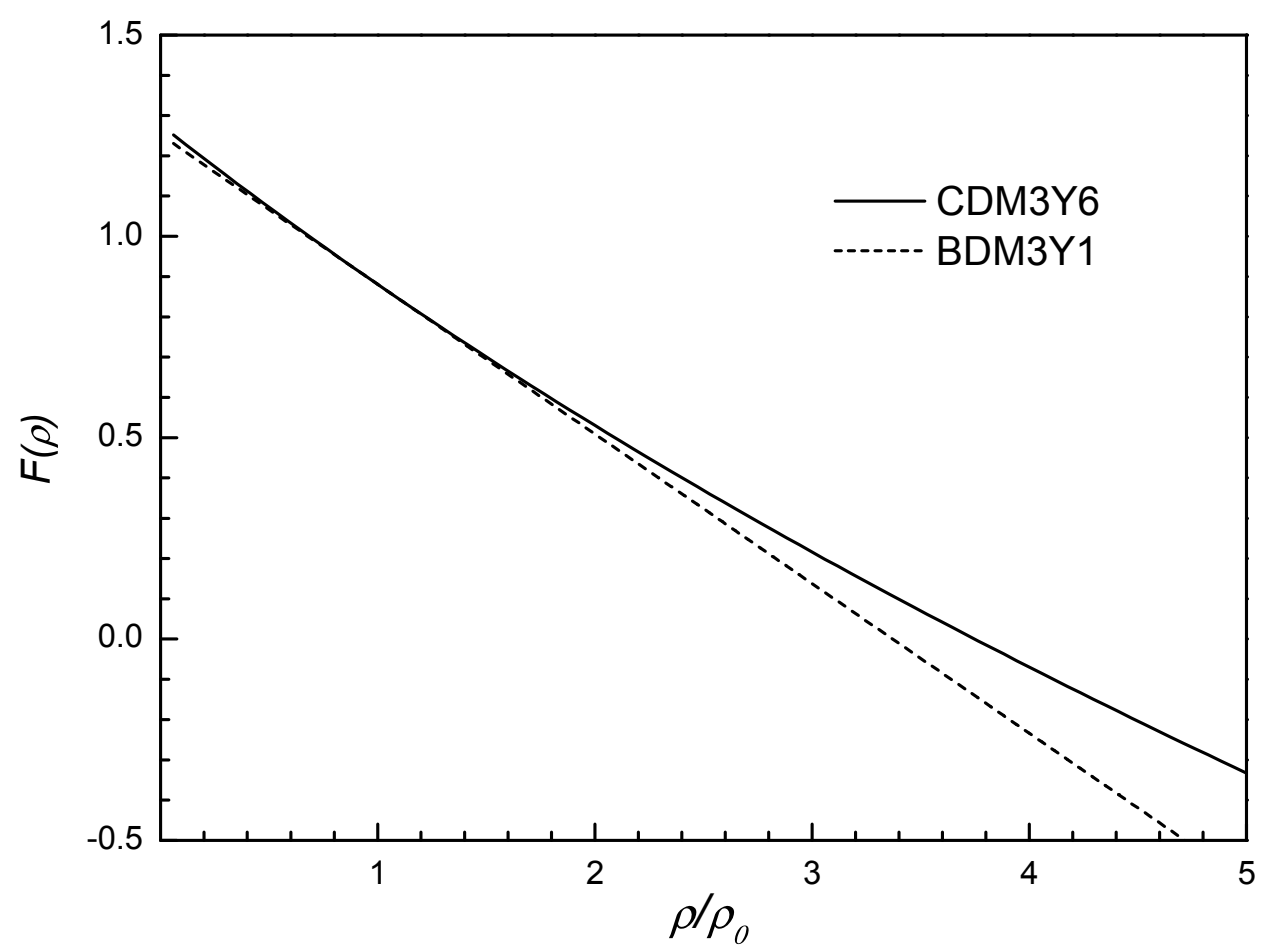

Fig. 1. The behavior of the density dependence BDM3Y1 and CDM3Y6 (see Eq. (4)) of the M3Y-Reid [11] and M3Y-Paris [12] interaction, respectively.

and M3Y-Paris [12] interactions, respectively, and parametrized $[8,10]$ as

$$
\begin{aligned}
& v_{\mathrm{D}(\mathrm{EX})}(E, \rho, s)=g(E) F(\rho) v_{\mathrm{D}(\mathrm{EX})}(s), \\
& \text { with } F(\rho)=C[1+\alpha \exp (-\beta \rho)-\gamma \rho] .
\end{aligned}
$$

The radial parts of the direct and exchange parts $v_{\mathrm{D}(\mathrm{EX})}(s)$ were kept unchanged, as derived from the original M3Y interactions, in terms of three Yukawas [11,12]. The explicit expressions of $v_{\mathrm{D}(\mathrm{EX})}(s)$, the linear energy dependent factor $g(E)$ and parameters $C, \alpha, \beta$ and $\gamma$ can be found, e.g., in Ref. [9]. The parameters (4) of the BDM3Y1 and CDM3Y6 density dependences have been carefully adjusted in the Hartree-Fock (HF) calculation to reproduce the saturation of the cold nuclear matter at $\rho=\rho_{0}$, with $\rho_{0} \approx 0.17 \mathrm{fm}^{-3}$, and give the nuclear matter incompressibility $K \approx 232$ and $252 \mathrm{MeV}$, respectively [9]. The behavior of the density dependent function $F(\rho)$ is shown in Fig. 1, and it has been probed quite accurately in the folding model analysis of the refractive $\alpha$-nucleus scattering, at the densities up to $\rho \approx 2 \rho_{0}[8,9]$. We note that the $\alpha$-particle has a very compact density that can be as high as $2 \rho_{0}$ in the center [19], and the static overlap density of the $\alpha+\alpha$ system may reach as much as $4 \rho_{0}$. Therefore, the use of a density dependent $\mathrm{NN}$ interaction in the folding calculation of the $\alpha+\alpha$ potential should be necessary. 
It should be noted that the BDM3Y1 and CDM3Y6 density dependences have been tailored in the HF calculation for a uniform nuclear matter that can be represented by a single Fermi sphere in the momentum space. The situation in a nucleus-nucleus collision is much more complicated, and the momentum distribution of the dinuclear system is a dynamic picture of two Fermi spheres separated by the local relative nucleon momentum. From the nuclear matter point of view, a realistic density dependent NN interaction for the folding model calculation of the nucleus-nucleus potential (or the dynamic simulation of the nucleus-nucleus collision based on a transport model) should be derived basically from a Brueckner-Hartree-Fock study of the two slabs of nuclear matter separated by different relative nucleon momenta, and at different asymmetries of the matter densities of the two slabs. Such an approach has been initiated in the past by Tuebingen group [20,21] but remains incomplete. It is, therefore, desirable that the issue raised in the present work will give a new motivation for such a microscopic study of dinuclear matter.

\section{II.2. Double-folding model}

The generalized DFM of Ref. [7] was used to evaluate the $\alpha+\alpha$ potential from the HF-type matrix elements (1) of the density-dependent interaction (3)-(4). The (local) direct term is readily evaluated by the standard double-folding integration

$$
U_{\mathrm{D}}(E, \boldsymbol{R})=\int \rho_{1}\left(\boldsymbol{r}_{1}\right) \rho_{2}\left(\boldsymbol{r}_{2}\right) v_{\mathrm{D}}(E, \rho, s) d^{3} r_{1} d^{3} r_{2}, \boldsymbol{s}=\boldsymbol{r}_{2}-\boldsymbol{r}_{1}+\boldsymbol{R} .
$$

The exchange term in Eq. (1) is generally nonlocal in the coordinate space, but a local form of the exchange potential can be obtained using the local WKB approximation [22] for the change in relative motion induced by the exchange of spatial coordinates of each interacting nucleon pair $[7,9]$

$$
\begin{array}{r}
U_{\mathrm{EX}}(E, \boldsymbol{R})=\int \rho_{1}\left(\boldsymbol{r}_{1}, \boldsymbol{r}_{1}+\boldsymbol{s}\right) \rho_{2}\left(\boldsymbol{r}_{2}, \boldsymbol{r}_{2}-\boldsymbol{s}\right) v_{\mathrm{EX}}(E, \rho, s) \\
\times \exp \left(\frac{i \boldsymbol{K}(\boldsymbol{R}) \boldsymbol{s}}{M}\right) d^{3} r_{1} d^{3} r_{2} .
\end{array}
$$

Here $\boldsymbol{K}(\boldsymbol{R})$ is the local momentum of relative motion determined from

$$
K^{2}(\boldsymbol{R})=\frac{2 \mu}{\hbar^{2}}\left[E-U(E, \boldsymbol{R})-V_{C}(\boldsymbol{R})\right]
$$

where $\mu$ is the reduced mass, $M=A_{1} A_{2} /\left(A_{1}+A_{2}\right) \equiv 2$ is the recoil factor or the reduced mass number, $E$ is the scattering energy in the center-of-mass frame, $U(E, \boldsymbol{R})$ and $V_{C}(\boldsymbol{R})$ are the nuclear and Coulomb parts of the $\alpha+\alpha$ potential, respectively. The calculation of $U_{\mathrm{EX}}$ is done iteratively using the explicit expression of the nonlocal density matrix given by the h.o. wave functions of nucleons bound in the two $\alpha$-particle [15]. To validate the folding model prediction for the nucleus-nucleus potential, it is important to discuss the treatment of the dinuclear (overlap) density embedded in the density dependence (4) of the M3Y interaction. In the present paper we consider three approximations for the $\alpha+\alpha$ overlap density 


\section{Frozen density approximation}

We recall that the DFM generates the first-order term of the microscopic OP in the Feshbach's scheme [23], used in the OM equation to obtain the relative-motion wave function of the two colliding nuclei being in their ground states. Given the antisymmetrization effects accurately taken into account via the exchange term (6), a reasonable approximation for the total density $\rho$ of the two overlapping nuclei is the sum of the two g.s. densities. In the calculation of the direct potential (5) the overlap density $\rho$ in $F(E, \rho)$ is taken as the sum of the two $\alpha$ densities at the position of each nucleon

$$
\rho=\rho_{1}\left(\boldsymbol{r}_{1}\right)+\rho_{2}\left(\boldsymbol{r}_{2}\right) .
$$

The assumption (8) was widely adopted in the DFM calculations with the density dependent NN interaction $[4,8-10,13-15]$ because it allows an explicit separation of variables in the three-dimensional integral (5). In evaluating the exchange potential (6), the overlap density in $F(\rho)$ is taken as the sum of the two $\alpha$ densities at the midpoint between the two nucleons being exchanged [7]

$$
\rho=\rho_{1}\left(\boldsymbol{r}_{1}+\frac{\boldsymbol{s}}{2}\right)+\rho_{2}\left(\boldsymbol{r}_{2}-\frac{\boldsymbol{s}}{2}\right) .
$$

The approximation (8)-(9), dubbed as frozen density approximation (FDA), has been used in most of the DFM calculations of the nucleus-nucleus potential using a density dependent NN interaction when the energy is not too low. Any density rearrangement that might happen during the collision would lead to the nuclear states different from the ground states, and thus contribute to higher-order dynamic polarization potential in the Feshbach's scheme [23]. The FDA reproduces very well the observed reduction of the attractive strength of the real nucleus-nucleus OP at small distances implied, in particular, by the refractive $\alpha$-nucleus scattering data $[9,15]$. The use of FDA has also been shown in the recent DFM calculations of the nucleus-nucleus OP at medium energies by RIKENOsaka group [24-26], using a realistic G-matrix interaction, as the most suitable for the nucleus-nucleus overlap density.

In the $\alpha+\alpha$ case, the static FDA gives the overlap density reaching $3 \sim 4 \rho_{0}$ at the smallest distances, and the considered density dependence of the M3Y interaction has not been tested at such a high density. Moreover, it is also questionable if a simple geometrical overlap of the two g.s. densities implied by the FDA is still a reasonable approximation for the density dependence (4) at very low energies. As noted above, a realistic density dependence of the effective NN interaction for the DFM calculation of the nucleus-nucleus potential should be, in general, constructed from a microscopic study of the dinuclear matter at different momentum separations and density asymmetries. In the momentum space, the distance separating the two colliding slabs of nuclear matter becomes small at low energies and the Pauli blocking should play a very crucial role in shaping the density dependence of the NN interaction in the dinuclear medium.

In addition to the FDA, other approximations have also been used for the overlap density in the DFM calculation like, e.g., the geometric or arithmetic averages of the two g.s. densities adopted [27] in the folding calculations using the JLM density dependent interaction [28]. 


\section{Fully antisymmetrized density of the $\alpha+\alpha$ system}

$\alpha+\alpha$ system is a very special case where one could check the validity of FDA for the overlap density by estimating explicitly the $\alpha+\alpha$ density from the fully antisymmetrized total wave function of 8 nucleons bound in the two $\alpha$ clusters. Using the microscopic cluster model suggested by Brink for ${ }^{8}$ Be resonance [29], the total density of the $\alpha+\alpha$ system at a given distance $R$ between the centres of mass of the two $\alpha$-particles can be determined as

$$
\rho(\boldsymbol{r}, R)=\left\langle\Psi(R)\left|\sum_{i=1}^{8} \delta\left(\boldsymbol{r}-\boldsymbol{r}_{i}\right)\right| \Psi(R)\right\rangle,
$$

where the total wave function $\Psi(R)$ of the system is determined as a Slater determinant of the single-particle wave functions of 8 nucleons bound in the two $\alpha$ clusters

$$
\Psi(R)=\mathcal{A}\left\{\psi_{\alpha_{1}} \times \psi_{\alpha_{2}}\right\} .
$$

Here $\mathcal{A}$ is the antisymmetrizer and $\psi_{\alpha_{1(2)}}$ is the antisymmetrized wave function built upon the single-particle wave functions of 4 nucleons bound in each $\alpha$ cluster. The single-particle wave functions are the same h.o. wave functions as those used to calculate the $\alpha$-density used in the present folding calculation. The details of the $\alpha+\alpha$ density calculation (10)(11) are given in the Appendix. We denote hereafter the use of the antisymmetrized overlap density in the density dependence $F(\rho)$ of the NN interaction as the AOD approximation.

The AOD procedure (see the next session and appendix) substantially changes the radial shape of the $\alpha+\alpha$ overlap density at small distances, and it is no more a direct sum of the two $\alpha$-densities. As can be seen from Eq. (23), the fully antisymmetrized density contains two remnants of the original $\alpha$-densities and an "interference" term that arises from the antisymmetrization of the 8-nucleon wave function. As a result, the simple geometrical overlap of the two $\alpha$-densities implied by the FDA does not hold any more if the full antisymmetrization is taken into account. We will consider in the next session how the AOD procedure affects the density dependence (4) of the M3Y interaction, and whether this helps to clarify the inconsistency of the DFM discussed in the introduction.

\section{Dynamic distortion of the FDA by Pauli blocking}

The AOD treatment is still a static approximation for the density dependence (4) of the M3Y interaction. To estimate the dynamic distortion of the density dependence by the Pauli blocking, we refer to the nuclear matter approach to study nucleus-nucleus OP by Tuebingen group [20,21], which has been improved and further developed by Soubbotin et al. [30]. Namely, a nucleus-nucleus collision can be locally considered as a collision of two slabs of nuclear matter whose densities are the local densities of the target and projectile. The momentum distribution of the dinuclear system with the given local densities $\rho_{1}$ and $\rho_{2}$ is represented by the two Fermi spheres with radii $k_{F_{1}}=\left(1.5 \pi^{2} \rho_{1}\right)^{1 / 3}$ and $k_{F_{2}}=$ $\left(1.5 \pi^{2} \rho_{2}\right)^{1 / 3}$, and their centres separated by the average momentum of the nucleon relative motion $k_{\text {rel }}$ (see Fig. 2). The original approach by Tuebingen group [20,21] has used $k_{\text {rel }}=$ $K_{\infty} / M$, where $K_{\infty}$ is derived from Eq. (7) at $R \rightarrow \infty$ and $M$ is the recoil factor in Eq. (6). However, the OP obtained in this nuclear matter approach has been found later to be out of the global systematics established for the nucleus-nucleus OP $[9,31]$. In the present 


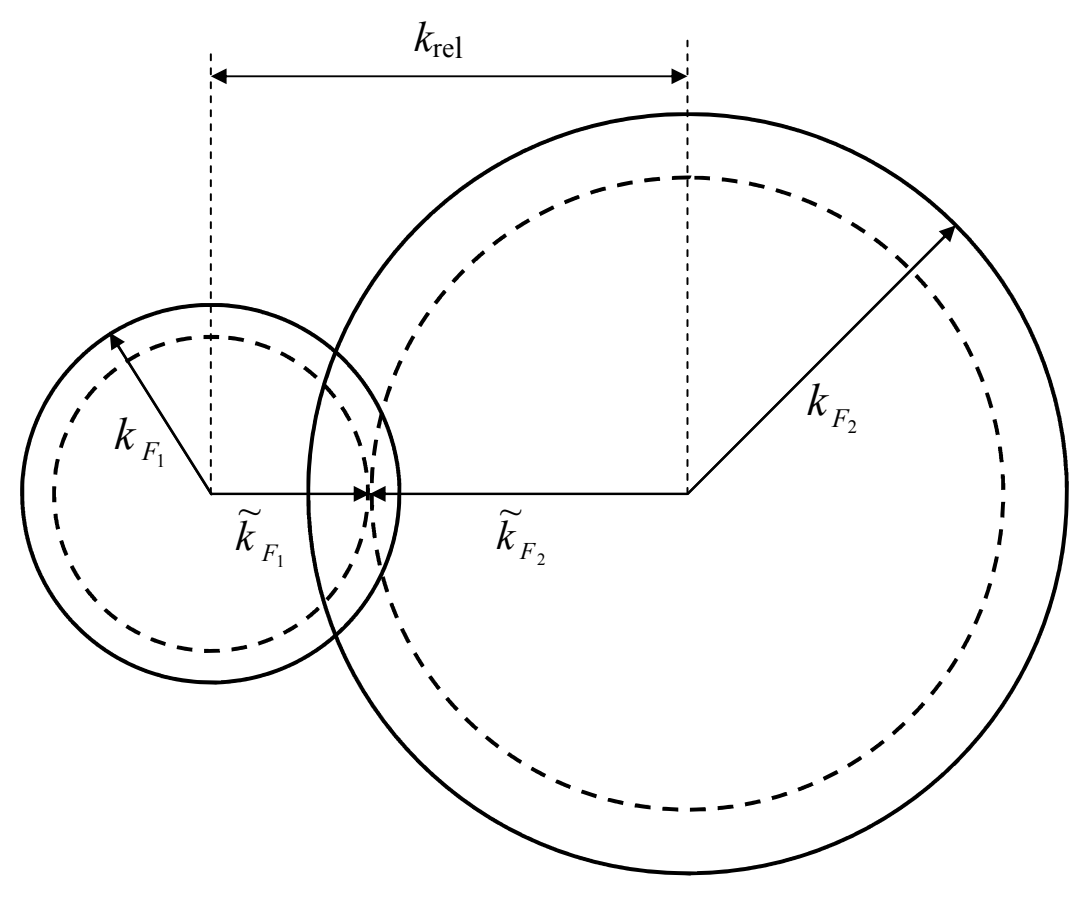

Fig. 2. The dynamic Pauli distortion of the two Fermi spheres representing the local densities of the two colliding nuclei in the momentum space.

study we have used the local relative-motion momentum of nucleon $k_{\text {rel }}(R)=K(R) / M$, with $K(R)$ determined self-consistently from the double-folded potential by Eq. (7). Such a treatment directly links the momentum distribution of the dinuclear density to the potential strength at each internuclear separation $R$, and it allowed to explain [30] the deep mean-field-type potential established by the global systematics [31] or predicted by the DFM [9]. The Pauli blocking forbids the overlap of the two Fermi spheres in the momentum space, and the shapes of the two Fermi spheres must be modified wherever $k_{F_{1}}+k_{F_{2}}>k_{\text {rel }}(R)$. In the nuclear matter approaches $[20,21,30]$ the OP between two nuclei separated by a distance $R$ is determined as the difference of the total energy of the dinuclear system at $R$ from that at infinite distance, based on the energy density formalism. Within this formalism, the total density $\left(\rho_{1}+\rho_{2}\right)$ must be unchanged and the Pauli distortion results, therefore, on a non-spherical shape of each distorted Fermi sphere (see, e.g., Fig. 1 in Ref. [30]). The non-spherical shapes of the two density distributions in the momentum space imply, however, that the two nuclei are no more in their ground states but in some (Pauli) excited states. As a result, the Pauli distorted total energy does not determine just the relative motion of the two nuclei being in their ground states, but the motion of a wave packet that includes also excited states. Such a wave packet is, in general, not appropriate for the description of elastic scattering [31]. 
To remain in the framework of the standard DFM $[7,8]$ without any hybrid coupling to the energy density formalism, we have suggested in the present work a procedure to improve the DFM by taking into account only the Pauli distortion of the density dependence (4) of the M3Y interaction. The densities entering the double-folding integration (5)-(6) remain unchanged, so that all the pair-wise interactions (1) between the projectile- and target nucleons are fully taken into account. Thus, the density dependent strength of the M3Y interaction is not static as adopted in the FDA, but is dynamically modified by the Pauli blocking at the small internuclear distances $R$. Whenever $k_{F_{1}}+k_{F_{2}}>k_{\text {rel }}(R)$, the corresponding local densities are reduced $\left(\rho_{1,2} \rightarrow \tilde{\rho}_{1,2}\right)$ so that the radii of the two reduced Fermi spheres satisfy relation $\tilde{k}_{F_{1}}+\tilde{k}_{F_{2}}=k_{\text {rel }}(R)$ that is allowed by the Pauli principle (see Fig. 2). The total density in (4) is reduced by such a "shrinkage" of the two local densities, but the projectile-target asymmetry $\chi$ is kept unchanged

$$
\chi=\frac{\rho_{1}}{\rho_{1}+\rho_{2}}=\frac{k_{F_{1}}^{3}}{k_{F_{1}}^{3}+k_{F_{2}}^{3}} \equiv \frac{\tilde{\rho}_{1}}{\tilde{\rho}_{1}+\tilde{\rho}_{2}}=\frac{\tilde{k}_{F_{1}}^{3}}{\tilde{k}_{F_{1}}^{3}+\tilde{k}_{F_{2}}^{3}} .
$$

Using condition (12) it is straightforward to obtain the reduced radii of the two distorted Fermi spheres as

$$
\tilde{k}_{F_{1}}=\frac{k_{\mathrm{rel}}}{1+X_{\chi}}, \tilde{k}_{F_{2}}=\frac{k_{\mathrm{rel}} X_{\chi}}{1+X_{\chi}}, \text { with } X_{\chi}=\left(\frac{1-\chi}{\chi}\right)^{\frac{1}{3}} .
$$

In difference from the nuclear matter approaches $[20,21,30]$, the two Fermi spheres distorted by the Pauli blocking remain spherical in this case (shown by dashed lines in Fig. 2). We denote hereafter the use of the overlap density modified by the dynamic Pauli distortion $\left(\rho=\tilde{\rho}_{1}+\tilde{\rho}_{2}\right)$ in Eq. (4) as the DPD approximation.

We stress again that, similar to the AOD procedure, the Pauli distorted local densities $\tilde{\rho}_{1,2}$ are used only to determine the overlap density used in the density dependence (4) of the M3Y interaction. The local and nonlocal nuclear densities entering the direct and exchange folding integrals (5)-(6) remain the original g.s. densities of the projectile and target, so that the DFM still determines the $\alpha+\alpha$ potential in the first order of the Feshbach's theory [23]. As can be seen from the discussion in the next section, the DPD procedure strongly reduces the dinuclear matter density $\left(\rho=\tilde{\rho}_{1}+\tilde{\rho}_{2}\right)$ entering the density dependence (4) of the M3Y interaction and helps to explain the observed depletion of the overlap $\alpha+\alpha$ density. On the other hand, the distorted total density is no more a direct sum of the two g.s. densities used in the double-folding integration (5)-(6), and this actually shows the breakdown of the FDA in the DFM description of the $\alpha+\alpha$ scattering at low energies.

\section{TREATMENT OF THE DENSITIES AND $\alpha+\alpha$ POTENTIAL}

The elastic $\alpha+\alpha$ scattering data are widely available at energies ranging from as low as $0.6 \mathrm{MeV}$ up to $\mathrm{GeV}$ region. In the present study we have considered only the data measured at the laboratory energies below the reaction threshold of $34.7 \mathrm{MeV}$, where the imaginary (absorptive) part of the OP is negligible and the elastic $\alpha+\alpha$ data can be well described by the real OP only. Different versions of the folded $\alpha+\alpha$ potential (1) 
were used in the present $\mathrm{OM}$ analysis of elastic $\alpha+\alpha$ data at energies of $E_{\alpha}=3$ to 29.5 $\mathrm{MeV}[32-36]$. To obtain the total OP, a Coulomb potential $V_{C}(R)$ needs to be added to the folded potential (1). In the OM studies of elastic nucleus-nucleus scattering, $V_{C}(R)$ is often chosen as the Coulomb potential between a point charge and a uniform charge distribution of the radius $R_{C}$. A more realistic choice of $V_{C}(R)$ for the nucleus-nucleus scattering is the Coulomb potential generated by double-folding two uniform charge distributions with radii $R_{C_{1}}=r_{C_{1}} A_{1}^{1 / 3}$ and $R_{C_{2}}=r_{C_{2}} A_{2}^{1 / 3}$ for which an analytic expression is available [37]. Both prescriptions give the correct asymptotic expression for the Coulomb potential,

$$
V_{C}(R) \rightarrow Z_{1} Z_{2} e^{2} / R
$$

but differ at small radii where the two nuclei overlap [31]. We have used in the present work the folded Coulomb potential [37] for the $\alpha+\alpha$ system, with the radii $r_{C_{1}}=r_{C_{2}}=1.36$ fm. All OM calculations were done using the direct reaction code PTOLEMY [38].

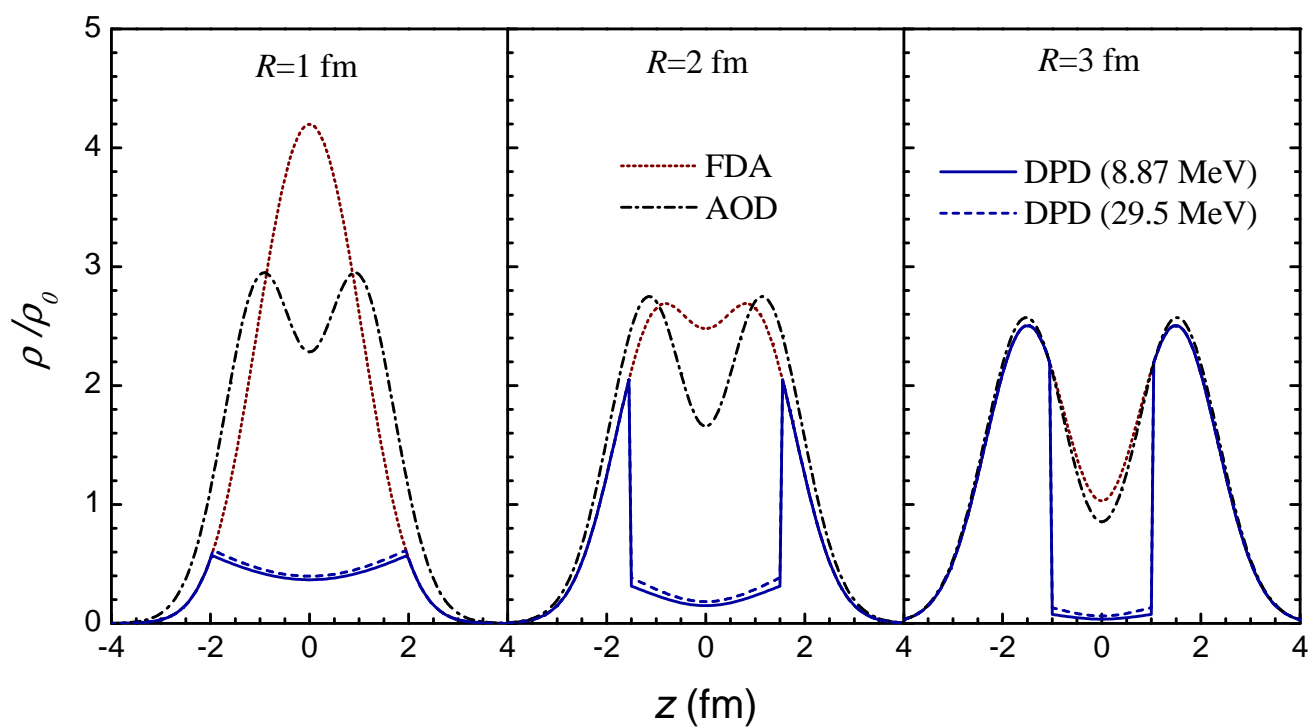

Fig. 3. The overlap $\alpha+\alpha$ density at different distances $R$ between the centres of the two $\alpha$-particles, given by different approximations for the density dependence of the CDM3Y6 interaction. The $z$-axis is aligned along the beam direction, with the origin at the center of mass of the $\alpha+\alpha$ system.

As noted above, the use of the density dependent NN interaction in the DFM analysis of the elastic $\alpha+\alpha$ scattering at low energies was not successful [14] when the FDA was used for the overlap density in the density dependence (4). The folded potential is usually too shallow in this case and fails to account for the elastic $\alpha+\alpha$ scattering data. The DFM can give a reasonable prediction of the $\alpha+\alpha$ potential only when the density dependence is neglected, i.e., to put $F(E, \rho)=1$ in Eq. (4) and use the original density independent M3Y interactions [11,12]. It is trivial to find out that this effect is due to a very high density profile of the ${ }^{4} \mathrm{He}$ nucleus. It is well established from the 


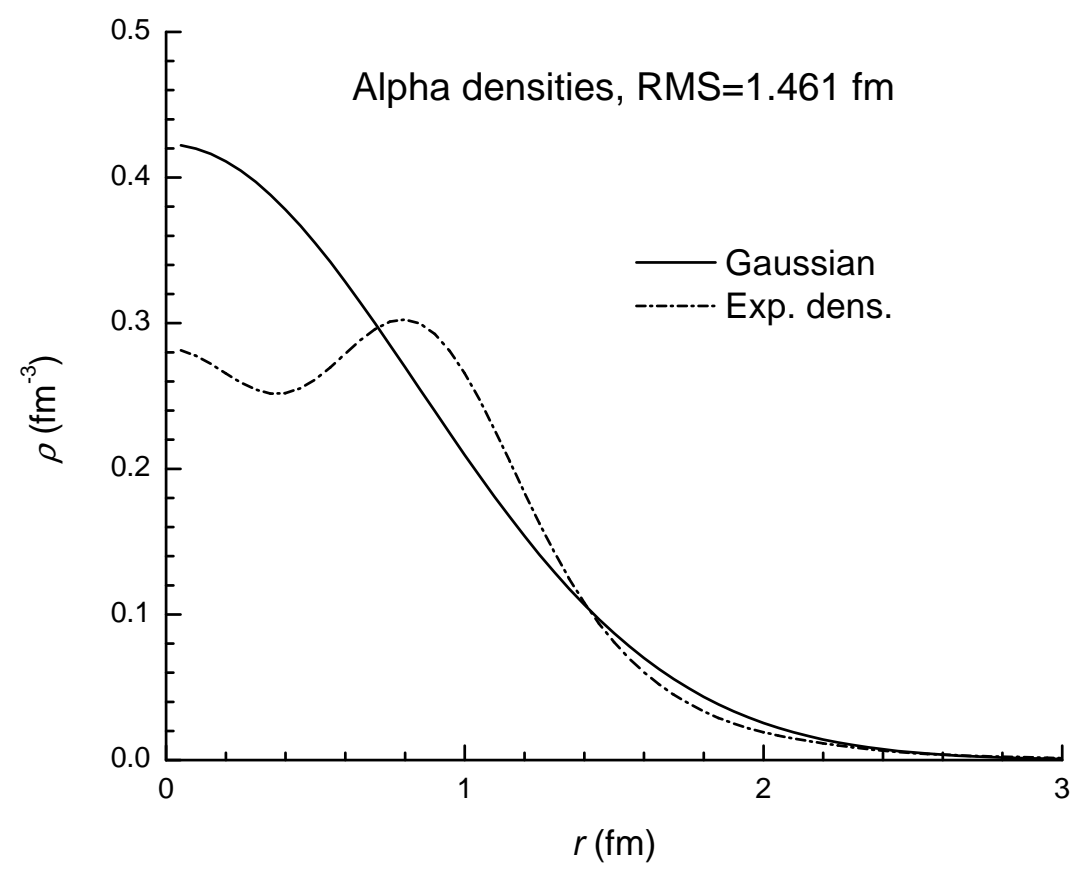

Fig. 4. The Gaussian $\alpha$-density (2) and the experimental $\alpha$-density (twice the experimental ${ }^{4} \mathrm{He}$ charge density [16] unfolded with the finite size of proton using the prescription of Ref. [4]). Both densities have the same RMS radius of $1.461 \mathrm{fm}$

electron scattering data [19] that the matter density of the $\alpha$-particle is $\rho \simeq 2 \rho_{0}$ around its center. As a result, the total density of the two $\alpha$-particles overlapping each other is reaching as much as $4 \rho_{0}$ in the FDA, and the folded $\alpha+\alpha$ potential becomes too shallow at small radii due to a strong repulsion caused by the steady decrease of $F(\rho)$ at high densities (see Fig. 1). The use of a more sophisticated, fully antisymmetrized overlap density (see Appendix) does not solve the problem. Although the overlap density is reduced in the center of the $\alpha+\alpha$ system by the full antisymmetrization (see Fig. 3), the folded potentials obtained with the AOD approximation are even slightly shallower than the folded potentials given by the FDA (see Fig. 5) due to a higher density given by AOD at the sub-surface region. It becomes obvious that at the low energies, the static overlap density of the $\alpha+\alpha$ system cannot be used in the density dependence (4) of the M3Y interaction, even when the antisymmetrization of the overlap density is taken into account exactly.

In a dynamic picture discussed in Sec. II, the Pauli blocking does not allow the overlap of the two Fermi spheres representing the local densities of the two $\alpha$-particles. At the considered energies, the distance between the two Fermi spheres, i.e., the nucleon relative momentum $k_{\text {rel }}(R)$ is quite small and reaches its maximum of $1.65 \sim 1.7 \mathrm{fm}^{-1}$ at the smallest radii $R$ (see Fig. 5). With the maximal radius $k_{F}$ of each Fermi sphere lying 


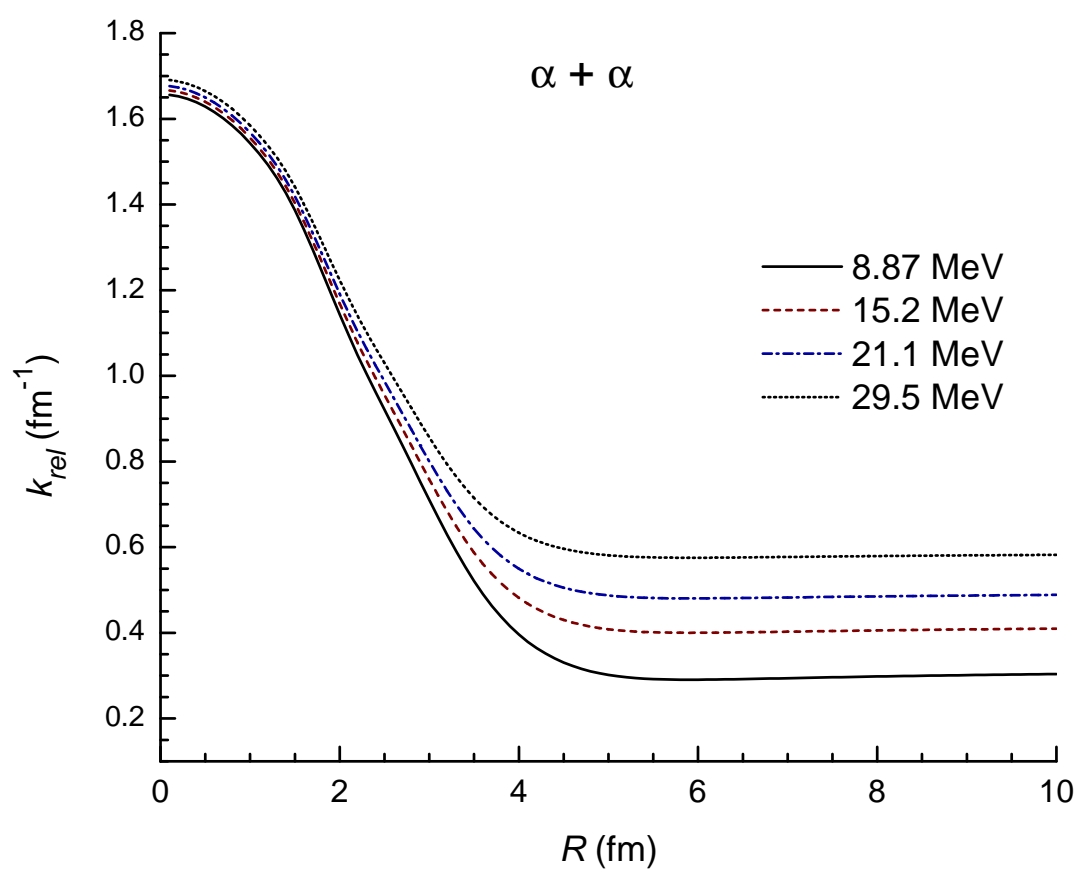

Fig. 5. The relative nucleon momentum $k_{\mathrm{rel}}=K(R) / M$ determined at different energies from the CDM3Y6 folded potential given by the DPD approximation for the density dependence.

around $1.70 \sim 1.75 \mathrm{fm}^{-1}$ (as given by the empirical $\alpha$ density [19]), the maximal $k_{\text {rel }}(R)$ value required by the Pauli blocking must be around $3.4 \sim 3.5 \mathrm{fm}^{-1}$ that is much larger than its maximum at the small distances. As a result, the two local densities entering the density dependence $F(\rho)$ should be strongly reduced by the dynamic Pauli distortion, $\tilde{\rho}_{1,2}<\rho_{1,2}$, as illustrated in Fig. 2. To implement the DPD procedure we check, at each distance $R$ between the centres of the two $\alpha$-particles, all possible overlaps of the two $\alpha$-densities in the coordinate space $\rho(\boldsymbol{r}, R)=\rho_{1}(\boldsymbol{r}, R)+\rho_{2}(\boldsymbol{r}, R)$, where $\boldsymbol{r}$ is the radius vector in the coordinate system with the origin at the center of mass and the $z$-axis lying along the beam direction as shown in Fig. 3. To prevent the self-contraction of a Fermi sphere having $k_{F}>k_{\text {rel }}(R)$ in the region where only one $\alpha$-density is dominated and the other $\alpha$-density is negligible, we have applied the DPD treatment only at the locations where both $\alpha$-particles have density $\rho \geqslant 0.005 \mathrm{fm}^{-3}$. At such a location the relation

$$
k_{F_{1}}+k_{F_{2}} \leqslant k_{\mathrm{rel}},
$$

is being checked and $\rho_{1,2}(\boldsymbol{r}, R)$ are replaced by $\tilde{\rho}_{1,2}(\boldsymbol{r}, R)$ using the prescription (13) wherever the relation (15) is not fulfilled. Such a DPD procedure is done iteratively until the radii of the two Fermi spheres always satisfy the relation (15). A lower limit of the $\alpha$-density automatically stops the DPD treatment at large distances $R$ where the two 


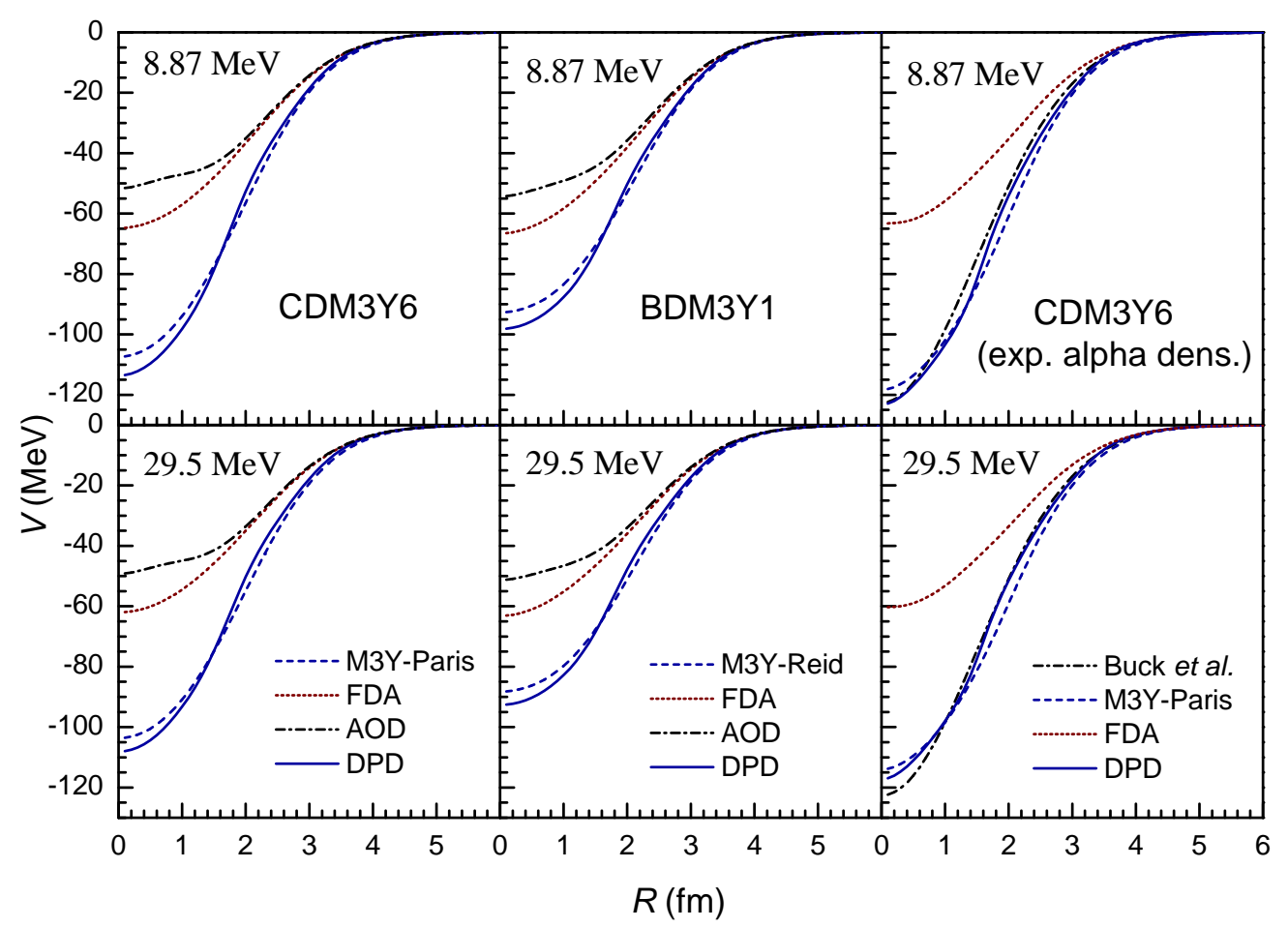

Fig. 6. The (unrenormalized) folded $\alpha+\alpha$ potentials at the incident energies $E_{\alpha}=8.87$ and $29.5 \mathrm{MeV}$ given by different approximations for the density dependence of the CDM3Y6 and BDM3Y1 interactions, in comparison with the (energy independent) Gaussian potentials (16) proposed by Buck et al. [3].

$\alpha$-particles are well separated and do not overlap in the coordinate space. This iterative DPD procedure consumes most of the CPU time in our dynamical double-folding calculation, which is about 3 orders of magnitude longer than that needed for the standard DFM calculation using the static overlap density given by the FDA. From the results shown in Fig. 3 one can see a substantial depletion of the central density at small distances resulted from the DPD treatment of the overlap density. At large distances $(R \geqslant 3 \mathrm{fm})$, the overlap of the two $\alpha$-particles in the coordinate space becomes less significant and the depletion of the overlap density occurs in a much smaller central spot. However, the density inside this central spot decreases very quickly, due to a fast drop of the $k_{\text {rel }}(R)$ values in the radial region $2 \mathrm{fm} \lesssim R \lesssim 4 \mathrm{fm}$ (see Fig. 5). Outside the central spot of the depleted density, the three approaches give about the same overlap density.

The folded $\alpha+\alpha$ potentials at $E_{\alpha}=8.87$ and $29.5 \mathrm{MeV}$ given by the three approximations for the density dependence (3-4) of the CDM3Y6 and BDM3Y1 interactions are shown in Fig. 6. A strong depletion of the central density by the DPD procedure leads readily to a much deeper (density dependent) potential that is comparable in strength and shape with the (density independent) M3Y-Paris and M3Y-Reid folded potentials. The folded potentials given by the M3Y-Reid interaction and its density dependent versions 
turned out to be slightly shallower than those given by the M3Y-Paris interaction. To be sure that the effects discussed here are not associated with a particular choice of the $\alpha$-density, we have used in our DFM calculation also the experimental $\alpha$-density, taken as twice the experimental ${ }^{4} \mathrm{He}$ charge density [16] unfolded with the finite size of proton using the prescription of Ref. [4]. The radial shapes of the two $\alpha$-densities are shown in Fig. 4 and some difference between them can be seen at small radii, although the two densities give the same RMS radius of $1.46 \mathrm{fm}$. The M3Y-Paris and CDM3Y6 folded potentials given by the experimental $\alpha$-density are plotted in the right panel of Fig. 6 , and the same drastic difference has been found in the results given by the FDA and DPD treatments of the density dependence of the M3Y-Paris interaction. In general, the folded potentials given by the experimental $\alpha$-density are somewhat deeper than those given by the Gaussian density (2).

\section{DESCRIPTION OF THE ELASTIC $\alpha+\alpha$ SCATTERING}

As mentioned above, the resonating group method $[3,5]$ is a rigorous approach that takes into account the full antisymmetrization of the total wave function of the $\alpha+\alpha$ system. It has been shown by Buck et al. [3] that about the same elastic $\alpha+\alpha$ phase shifts as well as other results of the full RGM calculation could be reproduced with an energy independent Gaussian potential

$$
V(R)=-V_{0} \exp \left(-\beta R^{2}\right)
$$

where $V_{0}=122.6225 \mathrm{MeV}$ and $\beta=0.22 \mathrm{fm}^{-2}$. The local potential (16) has been used quite successfully as the $\alpha-\alpha$ interaction in some cluster-folding calculations of the real OP between two $\alpha$-cluster nuclei $[39,40]$. The folded potentials at $E_{\alpha}=8.87$ and 29.5 $\mathrm{MeV}$ given by the experimental $\alpha$-density are compared with the Gaussian potential (16) in the right panel of Fig. 6, and one can see that the density independent M3Y-Paris and CDM3Y6 (DPD) potentials are quite close to the Gaussian potential at both energies. The folded CDM3Y6 (FDA) potential is too shallow and strongly differs from the Gaussian and CDM3Y6 (DPD) potentials at small radii.

The OM description of the elastic $\alpha+\alpha$ scattering data at $E_{\alpha}=8.87$ and 29.5 $\mathrm{MeV}$ given by the folded potentials discussed in Fig. 6 are shown in Fig. 7. From the left and middle panels of Fig. 7 one can see that the folded potentials obtained with the FDA and AOD approximations for the overlap density fail badly to account for the measured scattering cross sections. A reasonable description of the data is given by the folded potential obtained with the density independent M3Y interaction as found earlier in Ref. [14], but a much better description is given by the folded potential obtained with the density dependent CDM3Y6 or BDM3Y1 interaction and the DPD treatment of the density dependence. The OM results given by the folded potentials obtained with experimental $\alpha$-density are compared with those given by the Gaussian potential (16) of Buck et al. [3] in the right panel of Fig. 7. For the consistency, the Gaussian potential (16) has been used with the Coulomb potential $V_{C}(R)=4 e^{2} \operatorname{erf}(a R) / R$, with the $a$ value taken from Ref. [3]. From two versions of the folded potentials obtained with the DPD approximation, the CDM3Y6 potential (based on the M3Y-Paris interaction) accounts for the data better than the BDM3Y1 potential (based on the M3Y-Reid interaction). The 


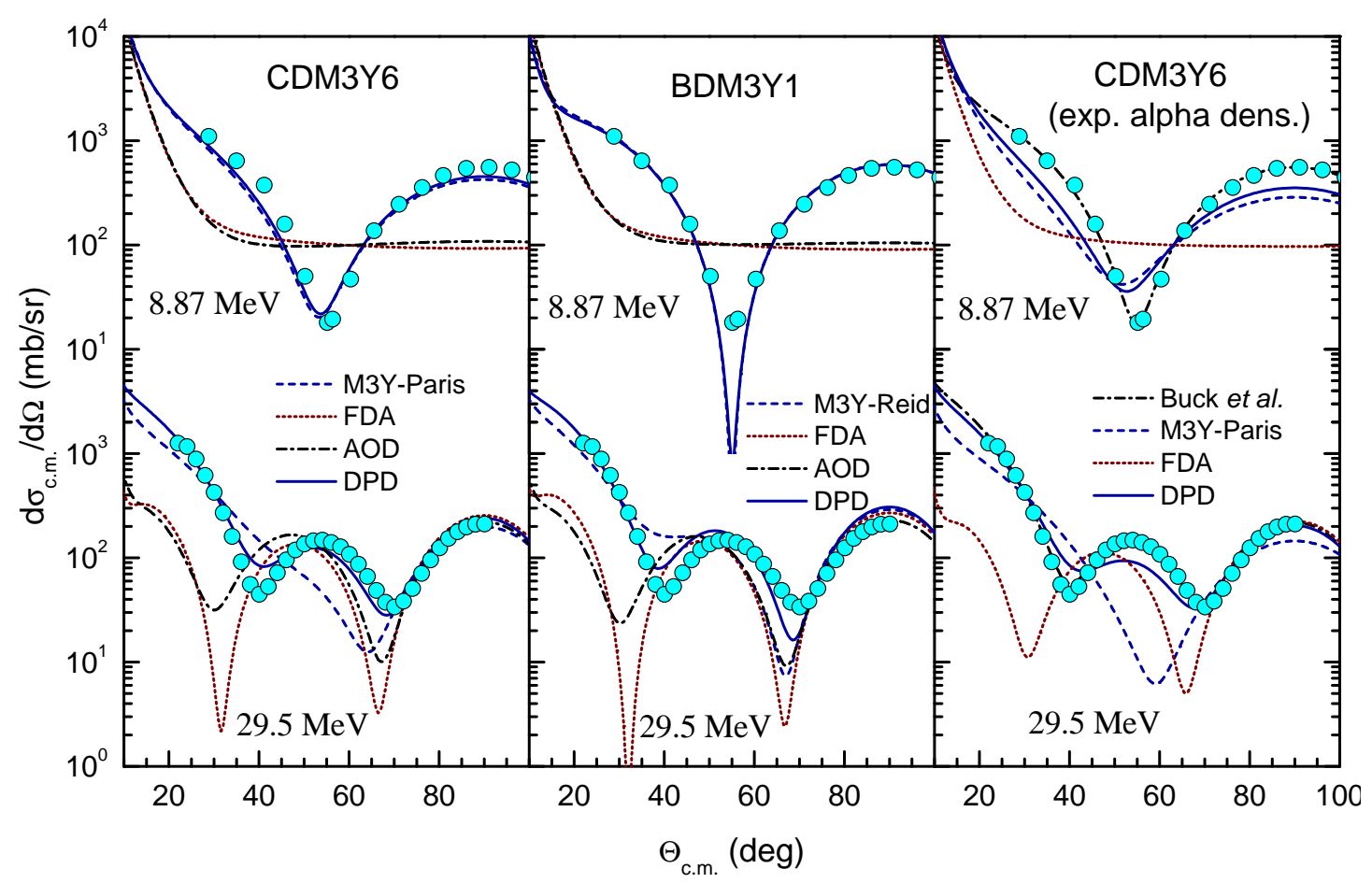

Fig. 7. The OM description of the elastic $\alpha+\alpha$ data measured at $E_{\alpha}=8.87$ and $29.5 \mathrm{MeV}$ given by the unrenormalized folded potentials obtained with different approximations for the density dependence of the CDM3Y6 (left panel) and BDM3Y1 (middle panel) interactions. The results given by the CDM3Y6 folded potentials obtained with the experimental $\alpha$-density are compared (right panel) with those given by the Gaussian potential of Buck et al. [3].

Gaussian potential of Buck et al. gives about the same good description of the data as that given by the CDM3Y6 potential obtained with the DPD approximation using the Gaussian density (2) for the $\alpha$-particle.

At the considered energies, when only the elastic channel is open and there is no coupling to other nonelastic channels, all the $\alpha+\alpha$ potentials under study should be used as given by the model, without any further renormalization of the potential strength. However, the $\alpha+\alpha$ potentials used in the present study are based on certain approximations and an adjustment (or renormalization) of the potential strength to the best OM fit of the data should be helpful in testing the potential model. We have made, therefore, also the $\mathrm{OM}$ calculation with different $\alpha+\alpha$ potentials, renormalizing the potential strength to obtain the best OM fit of the elastic data in each case. It is natural to expect that a realistic model for the $\alpha+\alpha$ potential should give its best OM description of the data with a renormalization coefficient $N$ close to unity. The model becomes less meaningful if $N$ strongly deviates from unity $[4,7,8]$. The OM results given by the renormalized $\alpha+\alpha$ potentials are compared with the data in Fig. 8, and the main properties of the 


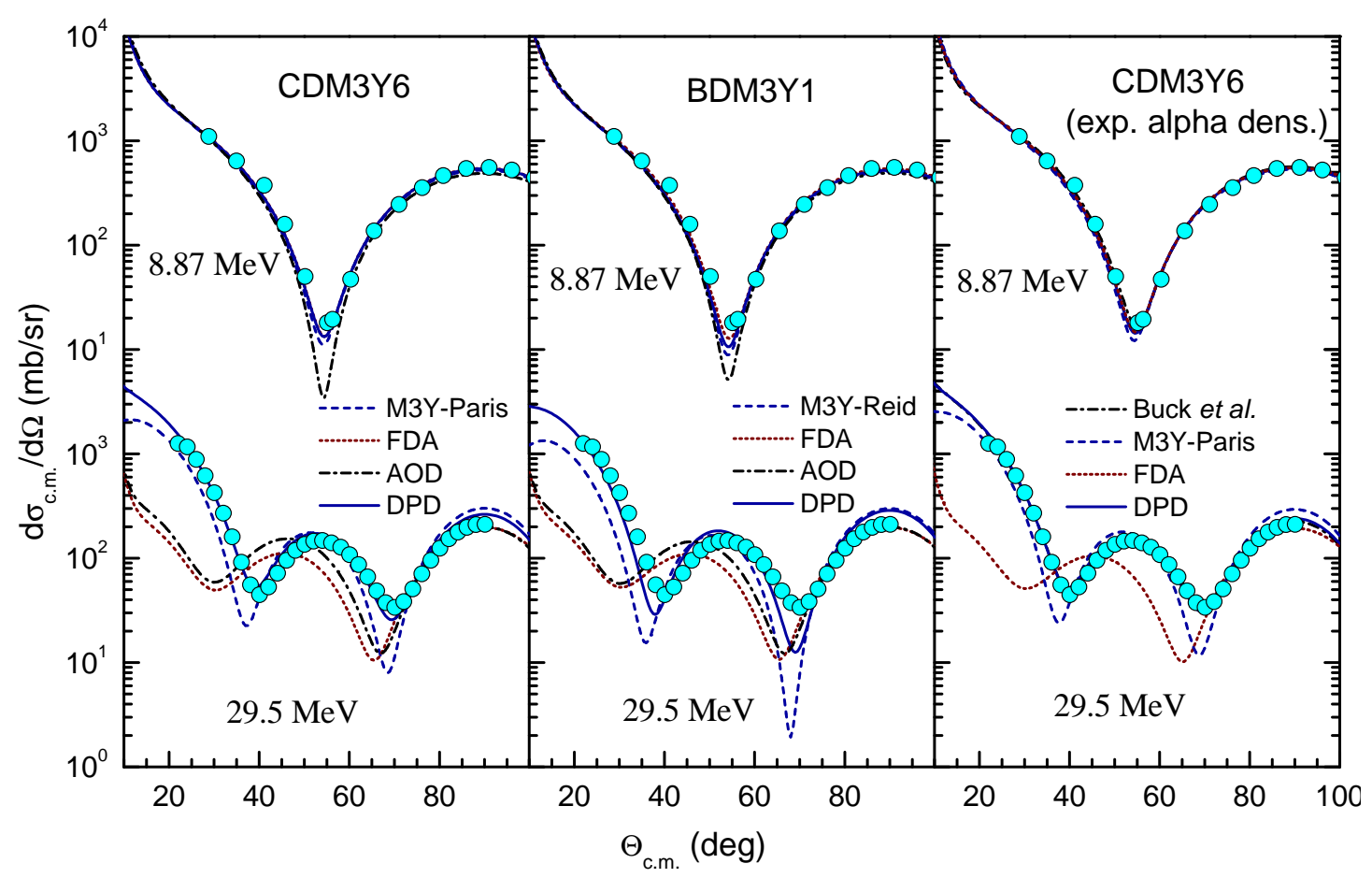

Fig. 8. The same as Fig. 7 but given by the renormalized folded and Gaussian potentials. See the renormalization coefficient $N$ and other properties of the $\alpha+\alpha$ optical potential in Table 1.

potential are given in Table 1. The low-energy data at $8.87 \mathrm{MeV}$ have a simple diffractive structure of one deep minimum that can be well described by all the folded potentials after some renormalization of the potential strength. However, one can see from Table 1 that the renormalization coefficients found for the folded potentials obtained with the FDA and AOD approximations are $N \approx 0.6$, much smaller than that found for the folded potential obtained with the DPD approximation $N \approx 0.96$. The $29.5 \mathrm{MeV}$ data have a more complicated oscillation structure that can be reproduced only by the Gaussian potential and the CDM3Y6 potential obtained with the DPD approximation. Without the absorption, the considered elastic $\alpha+\alpha$ scattering data are sensitive to the $\alpha+\alpha$ potential down to quite small impact parameter. As an illustration, the OM description of the 8.87 and $29.5 \mathrm{MeV}$ data given by the Gaussian potential of Buck et al., with and without the contribution of the $L=0$ partial wave, are plotted in Fig. 9. It is obvious that these data are quite sensitive to the partial wave $L=0$ that corresponds to the interaction distance of around $1 \mathrm{fm}$ between the two $\alpha$-particles (based on the semi-classical relation $L+1 / 2 \approx k R$, where $k$ is the wave number). At such short distances, the folded potentials obtained with the FDA and AOD approximations are too shallow and unable to account for the data at $29.5 \mathrm{MeV}$ even after a $\chi^{2}$-fit of the renormalization factor $N$. Given a 


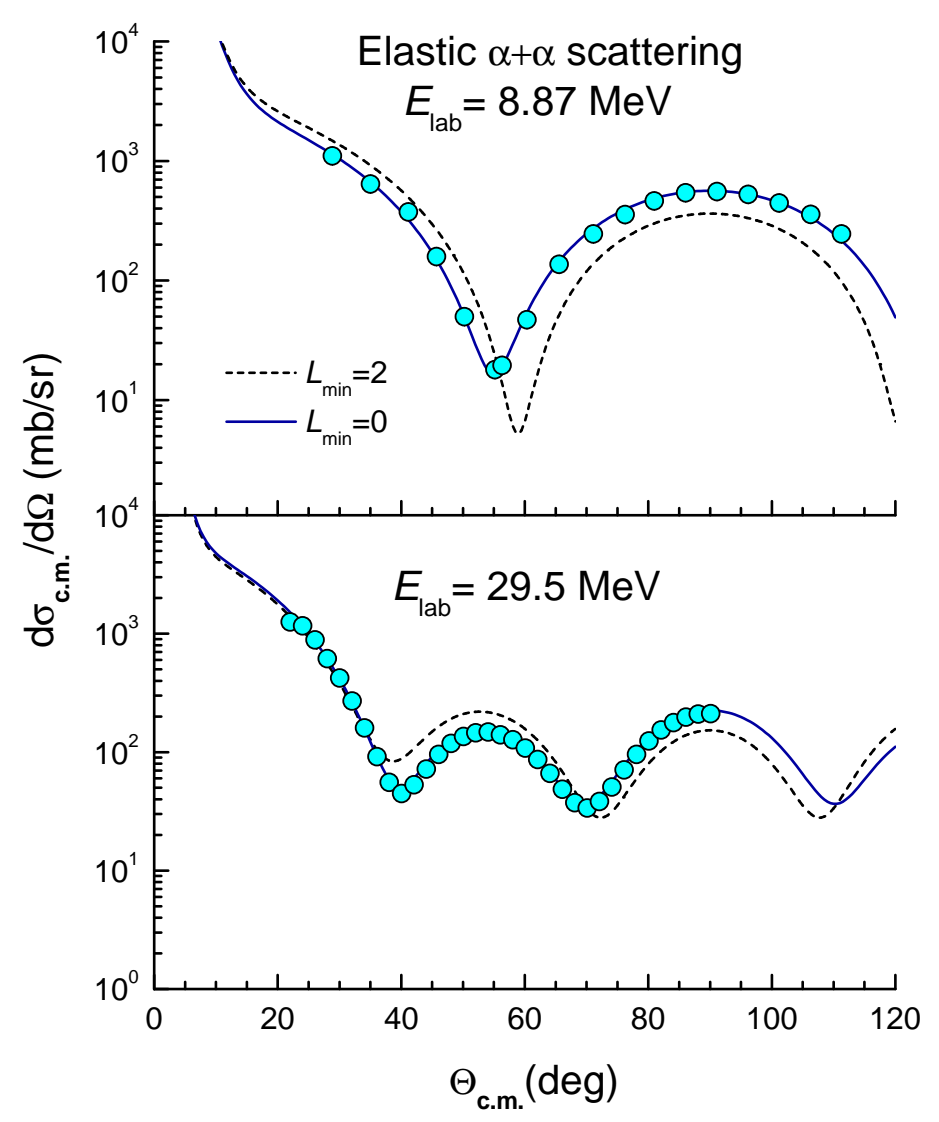

Fig. 9. The $\mathrm{OM}$ description of the elastic $\alpha+\alpha$ data measured at $E_{\alpha}=8.87$ and $29.5 \mathrm{MeV}$ given by the Gaussian potential of Buck et al. [3], with the lowest partial wave $L_{\min }=0$ and 2 .

best-fit $N$ coefficient quite close to unity that gives a very good OM description of both data sets, the CDM3Y6 potential obtained with the DPD approximation is undoubtedly the best prediction of the $\alpha+\alpha$ potential by the present folding model. The Gaussian potential of Buck et al. also gives a good description of the data at these energies, with the best-fit $N$ coefficient close to unity (see Table 1 ). A very important characteristic of the OP is the volume integral per interacting nucleon pair $J_{V}$ that has been often used to identify a given potential family [31]. It can be seen from Table 1 that the CDM3Y6 potential obtained with the DPD approximation for the density dependence turned out to have the best-fit $J_{V}$ value very close to that of the Gaussian potential of Buck et al.. These two potentials seem to belong to the same deep potential family (with $-J_{V} \approx 400$ $\mathrm{MeV} \mathrm{fm}{ }^{3}$ ) found from the global systematics of the light ion elastic scattering (see, e.g., Fig. 6.7 of Ref. [31]). The FDA and AOD approximations for the density dependence result on a too shallow folded potential whose $J_{V}$ value is significantly lower than that 
Table 1. The properties of the $\alpha+\alpha$ potentials at $E_{\alpha}=8.87$ and $29.5 \mathrm{MeV}$ that give the $\mathrm{OM}$ results shown in Fig. 8. $N$ is the renormalization coefficient of the potential found from the $\chi^{2}$-fit of the OM results to the elastic data. The folded potentials were obtained with the two choices of the $\alpha$-density, the Gaussian (2) and experimental density (see Fig. 4). $\chi^{2}$ value is per data point and obtained with uniform $10 \%$ errors.

\begin{tabular}{|c|c|c|c|c|c|c|}
\hline $\begin{array}{c}E_{\alpha} \\
(\mathrm{MeV})\end{array}$ & $\alpha$-density & Potential & $N$ & $\begin{array}{c}-J_{V} \\
\left(\mathrm{MeV} \mathrm{fm}^{3}\right)\end{array}$ & $\begin{array}{c}\left\langle r^{2}\right\rangle_{V}^{1 / 2} \\
(\mathrm{fm})\end{array}$ & $\chi^{2}$ \\
\hline 8.87 & - & Buck et al. & 0.996 & 412.0 & 2.611 & 0.6 \\
& $(2)$ & M3Y-Paris & 0.966 & 438.3 & 2.652 & 2.8 \\
& exp & M3Y-Paris & 0.902 & 435.2 & 2.645 & 2.2 \\
& $(2)$ & CDM3Y6 (DPD) & 0.971 & 419.7 & 2.618 & 1.7 \\
& exp & CDM3Y6 (DPD) & 0.935 & 415.1 & 2.615 & 1.2 \\
& $(2)$ & CDM3Y6 (FDA) & 0.587 & 187.5 & 2.777 & 1.5 \\
& exp & CDM3Y6 (FDA) & 0.611 & 187.8 & 2.796 & 1.1 \\
& $(2)$ & CDM3Y6 (AOD) & 0.628 & 190.1 & 2.811 & 8.8 \\
\hline 29.5 & - & Buck et al. & 0.990 & 409.6 & 2.611 & 0.6 \\
& $(2)$ & M3Y-Paris & 0.815 & 361.4 & 2.661 & 17.4 \\
& exp & M3Y-Paris & 0.784 & 368.2 & 2.652 & 13.2 \\
& $(2)$ & CDM3Y6 (DPD) & 0.958 & 396.5 & 2.627 & 3.3 \\
& exp & CDM3Y6 (DPD) & 0.949 & 401.8 & 2.621 & 1.2 \\
& $(2)$ & CDM3Y6 (FDA) & 0.889 & 272.7 & 2.785 & 34.8 \\
& exp & CDM3Y6 (FDA) & 0.922 & 271.0 & 2.805 & 34.5 \\
& $(2)$ & CDM3Y6 (AOD) & 0.959 & 279.5 & 2.820 & 46.0 \\
\hline
\end{tabular}

of the folded potential obtained with the DPD approximation. The fact that the folded potentials obtained with the FDA and AOD approximations could describe the $8.87 \mathrm{MeV}$ data well after being renormalized by $N \approx 0.6$ shows that the renormalized potentials have slipped into a very shallow potential family that gives a "phase-equivalent" description of elastic scattering at this energy. The shallow families of the $\alpha+\alpha$ potential were found long ago from the phase shift analysis of the low energy data [1], but these shallow potentials are empirical and cannot be associated with the results of a microscopic model like RGM or DFM.

For the simplicity, we discuss further only the DFM results obtained with the Gaussian density (2). The effects caused by different approximations for the density dependence to the DFM description of the elastic $\alpha+\alpha$ data at 8.87 and $29.5 \mathrm{MeV}$ were also confirmed in the $\mathrm{OM}$ analysis of the data at energies $3.0 \mathrm{MeV} \leqslant E_{\alpha} \leqslant 29.5 \mathrm{MeV}$, using the folded $\alpha+\alpha$ potentials. The elastic $\alpha+\alpha$ data plotted in Figs. 10 and 11 show clearly the evolution of the elastic $\alpha+\alpha$ angular distribution (with the increasing energy) from that having a single diffractive minimum to a more complicated oscillating structure with two pronounced diffractive minima. The (unrenormalized) folded potentials obtained with the 


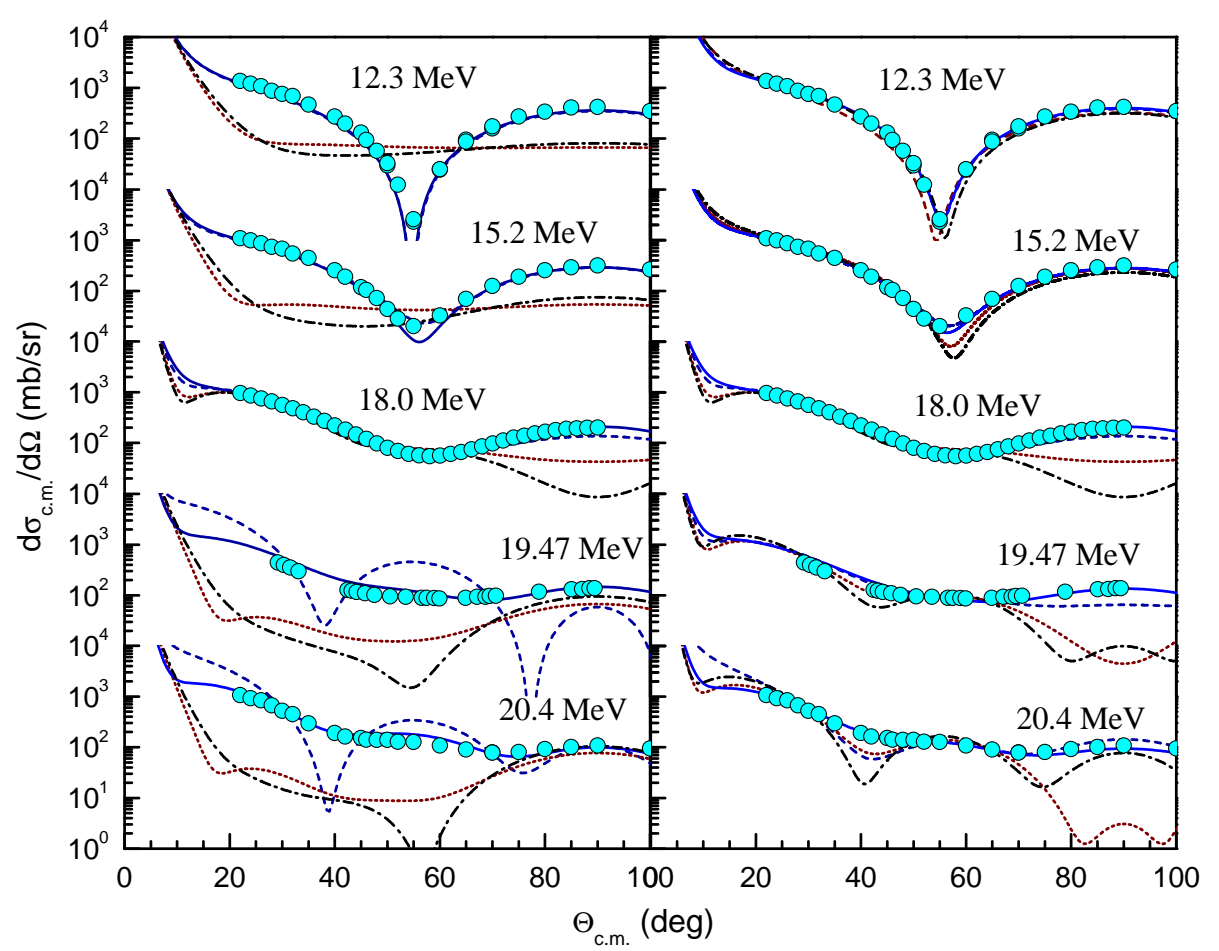

Fig. 10. The OM description of the elastic $\alpha+\alpha$ data at energies of $E_{\alpha}=12.3$ to $20.4 \mathrm{MeV}$ given by the unrenormalized (left panel) and renormalized (right panel) CDM3Y6 folded potentials obtained with different approximations for the density dependence. The notation of curves is the same as that in the left panel of Figs. 7 and 8.

FDA and AOD approximations fail completely to account for the data at all energies. After some renormalization of the potential strength, the single-minimum shape of the elastic cross section measured at low energies could be reproduced by all the folded potentials (see right panel of Fig. 10), but those given by the FDA and AOD approximations remain unable to describe the data at higher energies where the diffractive pattern is more complicated (see Fig. 11). The description of the data by the potential obtained with the density independent M3Y interaction also becomes worse with the increasing energy, and the only folded potential that gives consistently a good description of the data at all energies is that obtained with the density dependent M3Y interaction and DPD approximation for the density dependence. Given a renormalization coefficient $N \approx 0.96 \sim 0.98$, the CDM3Y6 potential obtained with the DPD approximation delivers a very good description of all the considered data (see right panels of Fig. 10 and 11). From the two density-dependent versions of the folded potential, the BDM3Y1 potential gives a slightly poorer fit to the data compared to that given by the CDM3Y6 potential (see Fig. 12). The best-fit $\mathrm{OM}$ results given by these two folded potentials are compared with those given 


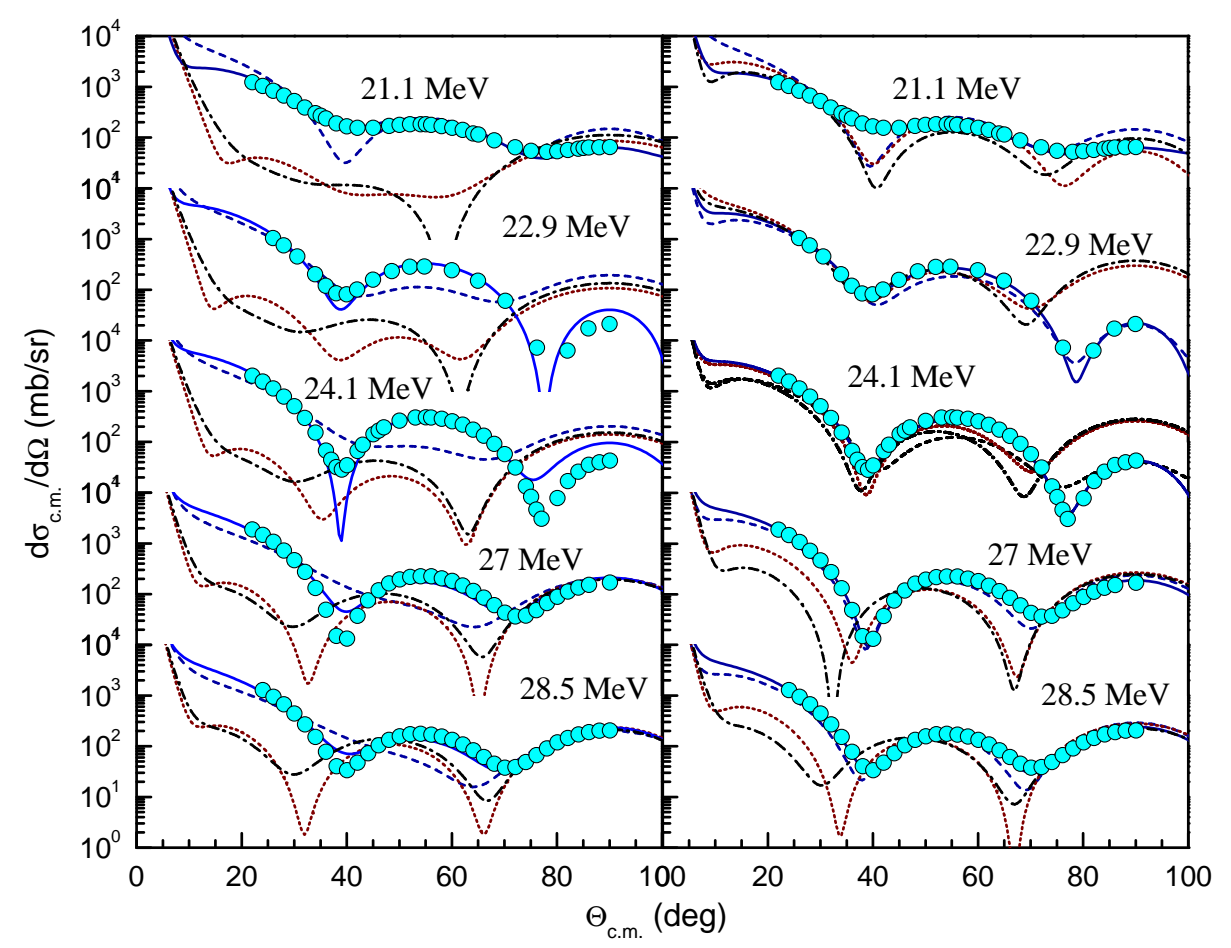

Fig. 11. The same as Fig. 10 but for the energies $E_{\alpha}=21.1$ to $28.5 \mathrm{MeV}$.

by the Gaussian potential of Buck et al. [3] in Fig. 12. With the Gaussian parameters adjusted to reproduce the $\alpha+\alpha$ resonance at the energy around $92 \mathrm{keV}$ and the elastic phase shift [3], the Gaussian potential gives an excellent OM description of the elastic $\alpha+\alpha$ data under study. One can see also from Fig. 13 that the best-fit renormalization factor $N$ of the Gaussian potential of Buck et al. is close to unity in most cases, except for the two energies of 12.3 and $15.2 \mathrm{MeV}$ where $N$ is falling below 0.95 . Thus, we conclude that the potential of Buck et al. [3] is a very realistic analytical form available for the local $\alpha+\alpha$ potential at low energies.

The best-fit renormalization factor $N$ of the CDM3Y6 (DPD) potential turned out to be also close to unity as shown in Fig. 13. The volume integrals per interacting nucleon pair $J_{V}$ of different folded potential are compared with those given by the Gaussian potential (16) in Fig. 14, and one can see that the CDM3Y6 (DPD) potential and the Gaussian potential belong indeed to the same potential family. Moreover, these two versions of the $\alpha+\alpha$ potential also give the best OM description of the elastic $\alpha+\alpha$ scattering data under study. Thus, we conclude that the DPD approximation is a much more accurate treatment of the density dependence (4) of the M3Y interaction compared to the FDA and AOD procedures. Although the DPD approximation leads to a very strong depletion of the overlap density in the center of the $\alpha+\alpha$ system as shown in Fig. 3, a total neglect 


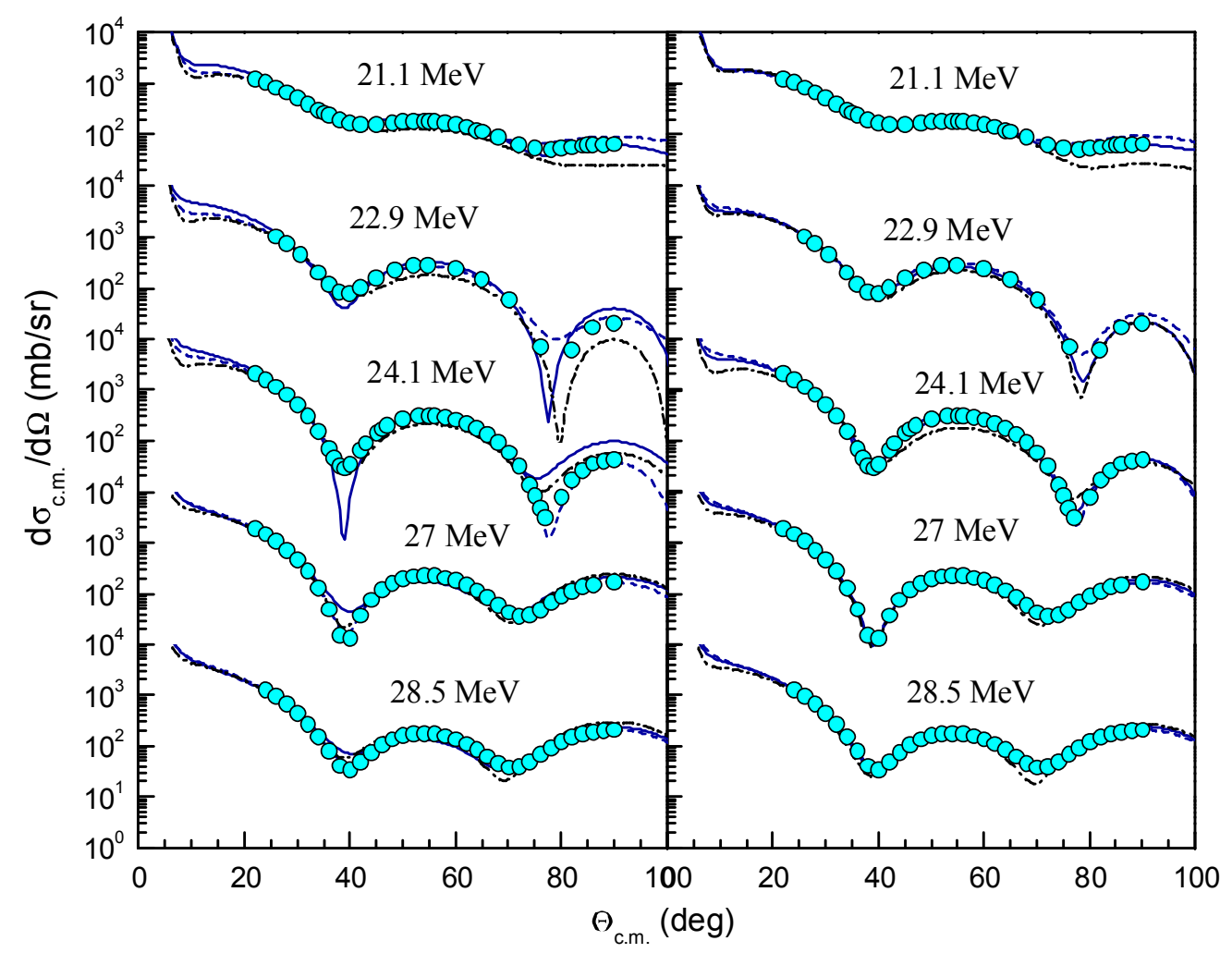

Fig. 12. The OM description of the elastic $\alpha+\alpha$ data at energies of $E_{\alpha}=21.1$ to $28.5 \mathrm{MeV}$ given by the unrenormalized (left panel) and renormalized (right panel) $\alpha+\alpha$ potentials. The results given by the CDM3Y6 and BDM3Y1 folded potentials obtained with the DPD approximation are shown as solid and dashdotted curves, respectively. The dash curves are the results given by the Gaussian potential of Buck et al. [3].

of the density dependence worsens somewhat the OM fit to the data, especially, the $\alpha+\alpha$ scattering data measured at energies around $20 \mathrm{MeV}$ and higher (see left panels of Figs. 10 and 11).

The present study has shown a vital role of the elastic scattering data measured over the whole observable angular range in testing different theoretical models for the $\alpha+\alpha$ potential. The elastic phase shifts deduced from the phase-shift analysis of these experimental cross sections are widely referred to in the literature as the experimental phase shifts. These quantities are, however, less sensitive to the detailed shape and strength of the potential compared to the measured elastic angular distribution. As illustration, the experimental elastic phase shift $\delta_{0}, \delta_{2}$ and $\delta_{4}$ at low energies [2] are compared with the OM results given by the Gaussian potential of Buck et al. [3] and the folded potentials in Fig. 15. One can see that the Gaussian potential gives a perfect fit to the experimental phase shifts at all energies, although the detailed OM analysis has shown (Fig. 13) that it 


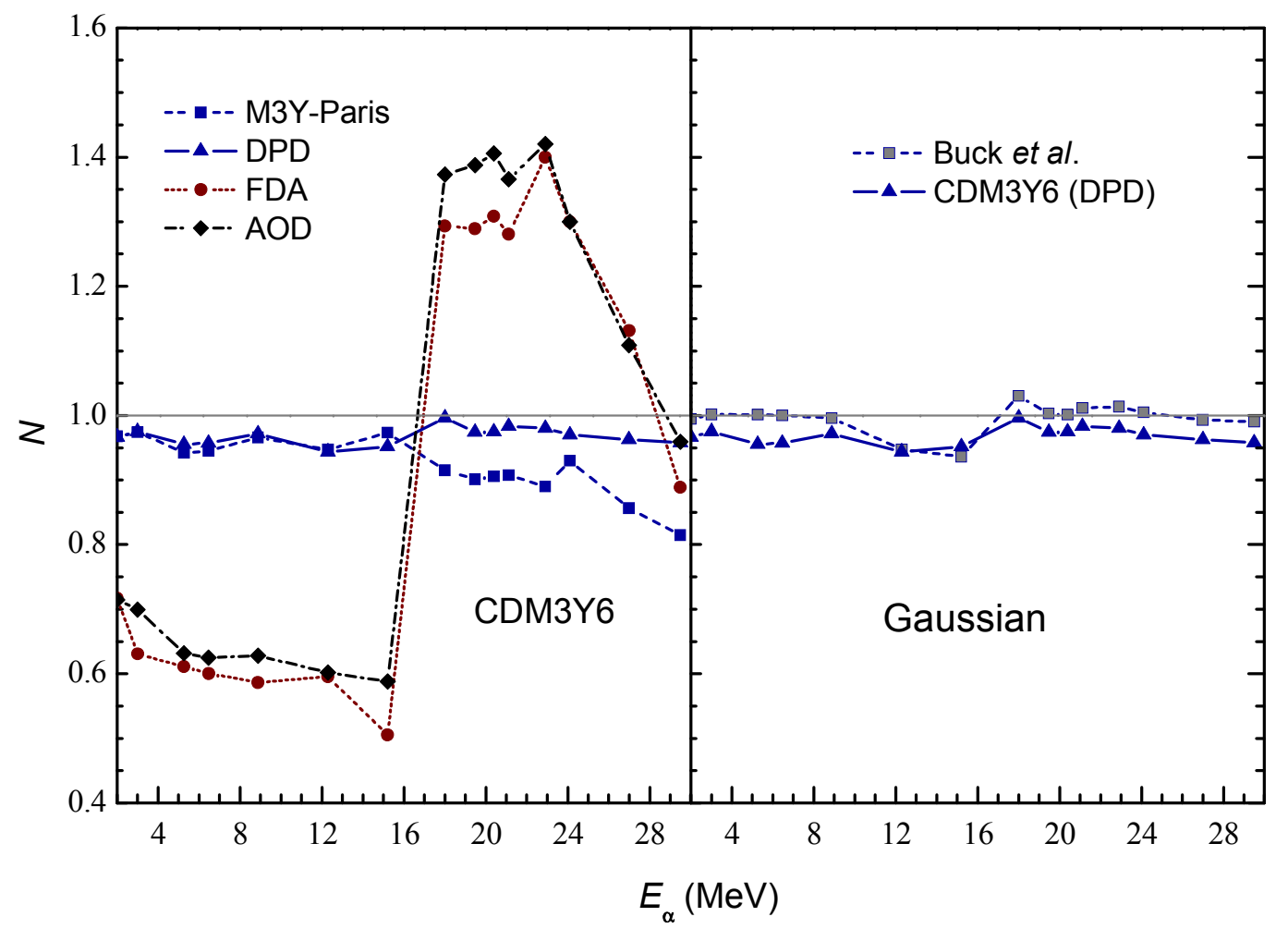

Fig. 13. The best-fit renormalization coefficient $N$ of the CDM3Y6 folded potentials obtained with different approximations for the density dependence and the Gaussian potential of Buck et al. [3] found by the OM analysis of the elastic $\alpha+\alpha$ data at energies of $E_{\alpha}=3$ to $29.5 \mathrm{MeV}$.

needs to be renormalized by a factor below 0.95 to fit the elastic data measured at energies of 12.3 and $15.2 \mathrm{MeV}$. The CDM3Y6 potential (obtained with the DPD approximation) renormalized by an average $N$ factor of 0.97 gives about the same good fit to the elastic phase shift, while the density independent M3Y-Paris potential (renormalized by an average $N$ factor of 0.94 ) fails to describe the phase shift data at energies above $20 \mathrm{MeV}$, in agreement with the OM results shown in Figs. 10 and 11.

\section{SUMMARY}

The OM analysis of the elastic $\alpha+\alpha$ scattering at energies below the reaction threshold of $34.7 \mathrm{MeV}$ has been done using the folded potentials obtained with the CDM3Y6 and BDM3Y1 versions of the density dependent M3Y interaction $[8,10]$. Different approximations for the density dependence (4) of the chosen interactions have been tested in the OM calculation and the results were compared with those given by the Gaussian potential of Buck et al. [3] that is based on the microscopic RGM results. The elastic $\alpha+\alpha$ data, in terms of the elastic scattering cross section measured accurately over the 


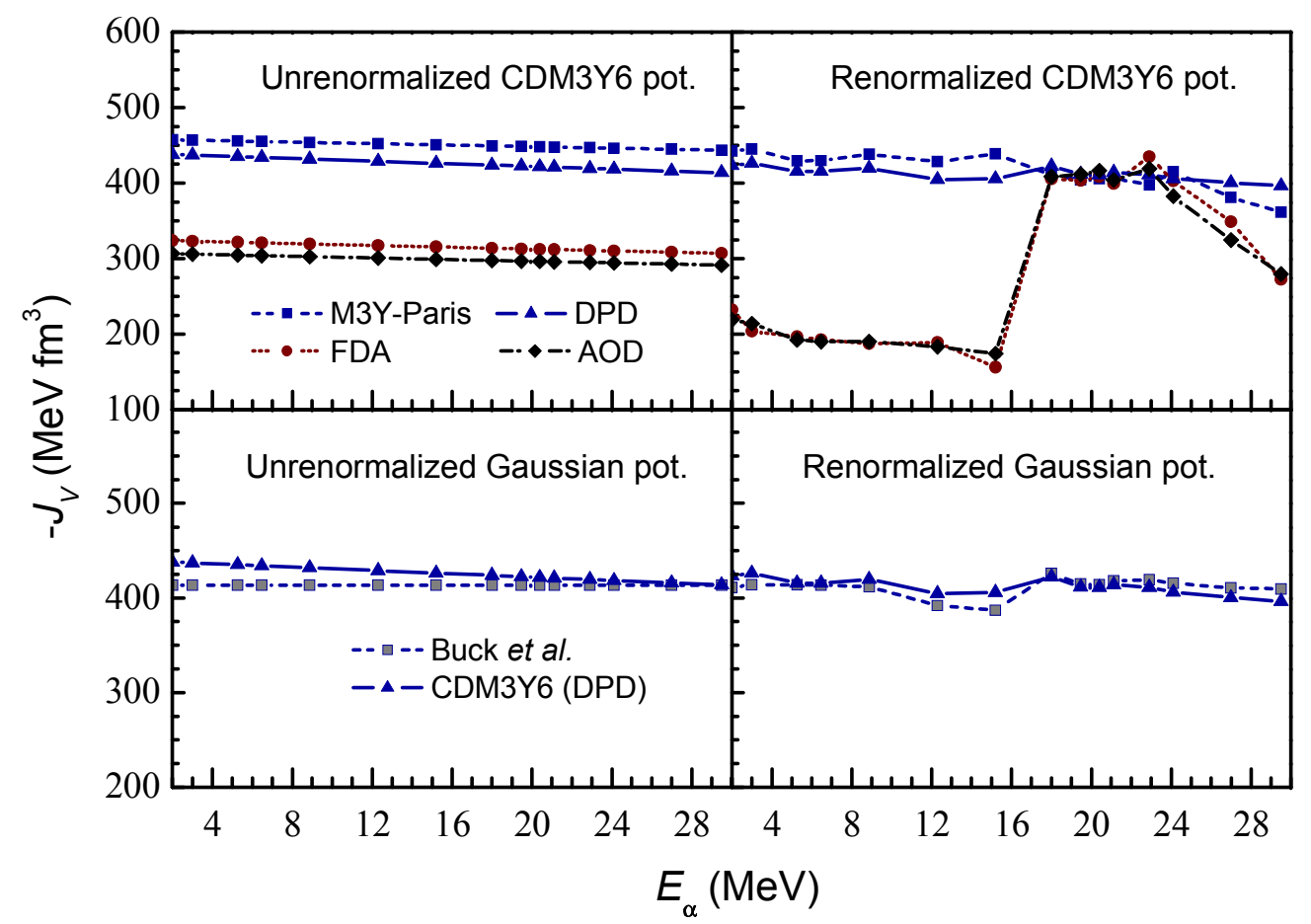

Fig. 14. The volume integral per interacting nucleon pair $J_{V}$ of the unrenormalized (left panel) and renormalized (right panel) CDM3Y6 folded potentials obtained with different approximations for the density dependence and the Gaussian potential of Buck et al. [3]. The $J_{V}$ values in the right panel are given by the $\alpha+\alpha$ potentials renormalized by the corresponding $N$ coefficients shown in Fig. 13 .

whole observable angular range, were shown to be a very efficient test ground for different theoretical models of the $\alpha+\alpha$ potential.

Our consistent folding model description of the elastic $\alpha+\alpha$ data under study has shown that the DPD approximation, based on the dynamic Pauli distortion of the two overlapping $\alpha$-densities in the momentum space, gives the best folding model prediction of the $\alpha+\alpha$ potential at low energies. The CDM3Y6 folded potential obtained with the DPD approximation turned out to be very close in strength and shape to the Gaussian potential of Buck et al. that was constructed to reproduce the experimental elastic $\alpha+\alpha$ phase shifts and the RGM results for ${ }^{8}$ Be resonance [3]. These two potentials were shown to belong to the same potential family in terms of the potential depth and volume integral. They also give equally good description of the elastic $\alpha+\alpha$ data under study. The present OM analysis of the elastic $\alpha+\alpha$ scattering has also confirmed that the Gaussian potential of Buck et al. is a reliable analytical expression for the local $\alpha+\alpha$ optical potential at low energies. This result validates, therefore, the use of this Gaussian potential as the $\alpha-\alpha$ interaction in the $\alpha$-cluster folding calculation $[39,40]$. 


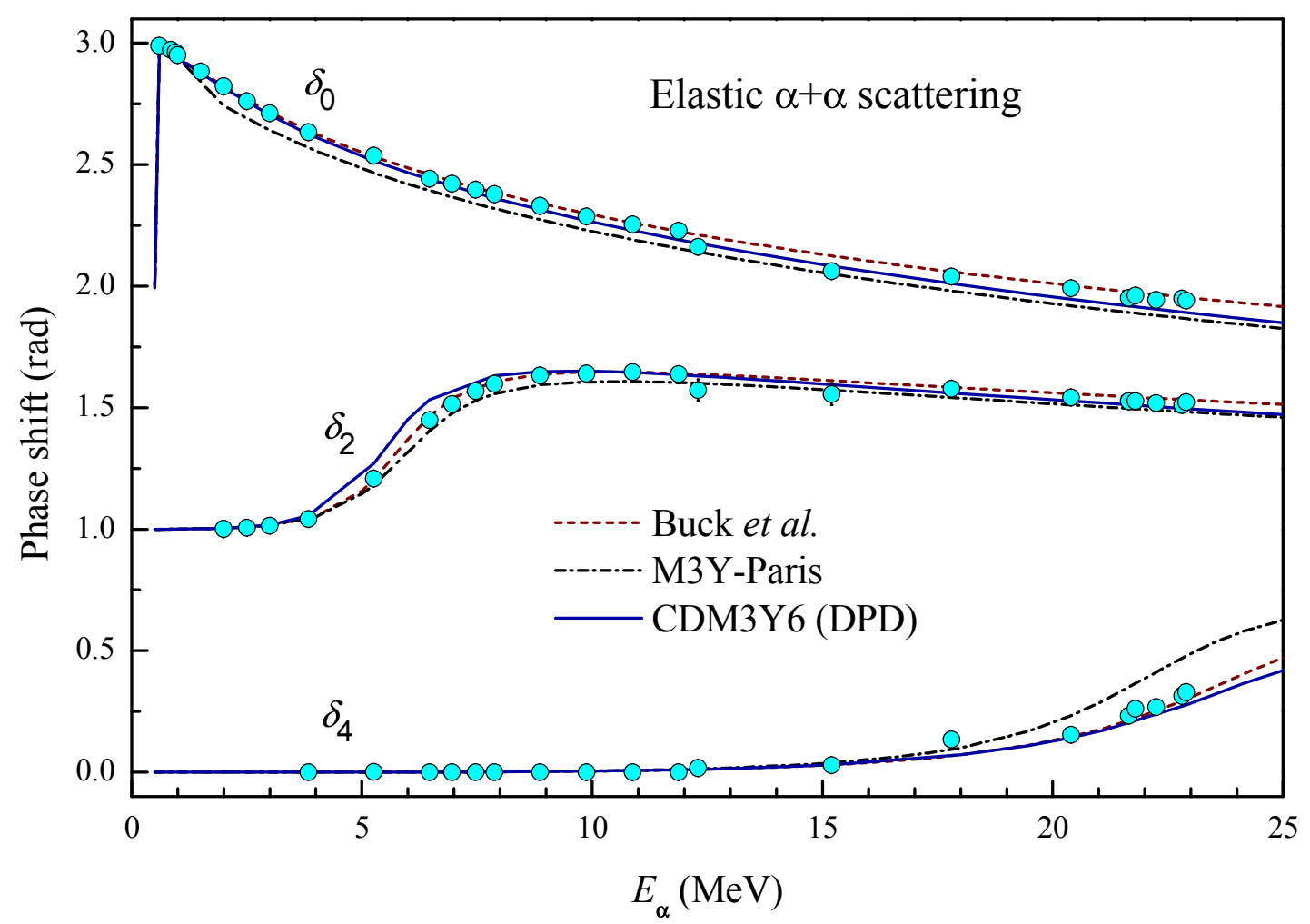

Fig. 15. The experimental elastic $\alpha+\alpha$ phase shift $\delta_{0}, \delta_{2}$ and $\delta_{4}$ at low energies [2] in comparison with the OM results given by the (unrenormalized) Gaussian potential of Buck et al. [3] and the folded potentials obtained with the density independent M3Y-Paris interaction and density dependent CDM3Y6 interaction (using the DPD approximation). The M3Y-Paris and CDM3Y6 folded potentials are renormalized by $N=0.94$ and 0.97 , respectively.

Although the DPD treatment of the density dependence (4) of the M3Y interaction is just a local approximation based on the Pauli blocking of the overlap of the two $\alpha$ densities in a dinuclear matter picture in the momentum space, the results of the present study show clearly that the density dependence of the effective NN interaction should be strongly distorted at the small distances between the two $\alpha$-particles. This results seems to explain a long standing problem of the DFM. Namely, the failure of the DFM in a consistent description of both the $\alpha+\alpha$ and $\alpha$-nucleus optical potentials at low energies using the same realistic density dependent $\mathrm{NN}$ interaction is due to a breakdown of the FDA by the Pauli blocking. The DPD approximation for the density dependence of the M3Y interaction can also be applied to study different nucleus-nucleus systems within the same dynamic DFM approach, and it is expected to improve the performance of the DFM 
at low energies, near the Coulomb barrier. We plan to carry out this topical research in the near future.

\section{ACKNOWLEDGMENTS}

The authors thank Yoshiko Kanada-En'yo for her helpful communication. The present research has been supported by the National Foundation for Science \& Technology Development (NAFOSTED project No. 103.04-2011.21) and by the LIA program of the Ministry of Science and Technology (MOST).

\section{REFERENCES}

[1] S. Ali and A.R. Bodmer, Nucl. Phys. 80 (1966) 99.

[2] S. A. Afzal, A. A. Z. Ahmad, and A. Ali, Rev. Mod. Phys. 41 (1969) 247.

[3] B. Buck, H. Friedrich, and C. Wheatley, Nucl. Phys. A 275 (1977) 246.

[4] G. R. Satchler and W. G. Love, Phys. Rep. 55 (1979) 183.

[5] H. Horiuchi, Nucl. Phys. A 522 (1991) 257; H. Horiuchi, in Trends in Theoretical Physics, eds. P.J. Ellis and Y.C. Tang, Addison-Wesley, New York, 1991.

[6] J. P. Day, J. E. McEwen, M. Elhanafy, E. Smith, R. Woodhouse, and Z. Papp, Phys. Rev. C 84 (2011) 034001.

[7] D. T. Khoa and G. R. Satchler, Nucl. Phys. A 668 (2000) 3.

[8] D. T. Khoa, G. R. Satchler, and W. von Oertzen, Phys. Rev. C 56 (1997) 954.

[9] D. T. Khoa, W. von Oertzen, H. G. Bohlen, and S. Ohkubo, J. Phys. G 34(2007) R111.

[10] D.T. Khoa and W. von Oertzen, Phys. Lett. B 342 (1995) 6.

[11] G. Bertsch, J. Borysowicz, H. McManus, and W. G. Love, Nucl. Phys. A 284 (1977) 399.

[12] N. Anantaraman, H. Toki, and G. F. Bertsch, Nucl. Phys. A 398 (1983) 269.

[13] A. M. Kobos, B. A. Brown, P. E. Hodgson, G. R. Satchler, and A. Budzanowski, Nucl. Phys. A 384 (1982) 65; A. M. Kobos, B. A. Brown, R. Lindsay, and G. R. Satchler, Nucl. Phys. A 425 (1984) 205.

[14] M. Avrigeanu, W. von Oertzen, A. J. M. Plompen, and V. Avrigeanu, Nucl. Phys. A 723 (2003) 104.

[15] D. T. Khoa, Phys. Rev. C 63 (2001) 034007.

[16] C. W. De Jager, H. De Vries, and C. De Vries, At. Data Nucl. Data Tables 14 (1974) 479.

[17] H. De Vries, C. W. De Jager, and C. De Vries, At. Data Nucl. Data Tables 36 (1987) 495.

[18] L. J. Tassie and F. C. Barker, Phys. Rev. 111 (1958) 940.

[19] I. Sick, J. S. McCarthy, and R. R. Whitney, Phys. Lett. B 64 (1976) 33.

[20] N. Ohtsuka, R. Linden, A. Faessler, and F. B. Malik, Nucl. Phys. A 465 (1987) 550, and references therein.

[21] D. T. Khoa, N. Ohtsuka, and A. Faessler, J. Phys. G 16 (1990) 1253.

[22] G.R. Satchler, Direct Nuclear Reactions, Clarendon Press, Oxford, 1983.

[23] H. Feshbach, Theoretical Nuclear Physics Volume II, Wiley-Interscience, New York, 1992.

[24] T. Furumoto, Y. Sakuragi, and Y. Yamamoto, Phys. Rev. C 80 (2009) 044614.

[25] T. Furumoto, Y. Sakuragi, and Y. Yamamoto, Phys. Rev. C 82 (2010) 044612.

[26] T. Furumoto, W. Horiuchi, M. Takashina, Y. Yamamoto, and Y. Sakuragi, Phys. Rev. C 85 (2012) 044607.

[27] L. Trache, A. Azhari, H. L. Clark, C. A. Gagliardi, Y. W. Lui, A. M. Mukhamedzhanov, R. E. Tribble, and F. Carstoiu, Phys. Rev. C 61 (2000) 024612.

[28] J. P. Jeukenne, A. Lejeune, and C. Mahaux, Phys. Rev. C 16 (1977) 80.

[29] H. Horriuchi, Lecture Notes in Physics vol. 818, 57 (2010), Springer-Verlag Berlin Heidelberg, 2010.

[30] V. B. Soubbotin, W. von Oertzen, X. Vinas, K. A. Gridnev, and H. G. Bohlen, Phys. Rev. C 64 (2001) 014601.

[31] M. E. Brandan and G.R. Satchler Phys. Rep. 285 (1997) 143. 
[32] N. P. Heydenburg and G. M. Temmer, Phys. Rev. 104 (1956) 123.

[33] T. A. Tombrello and L. S. Senhouse, Phys. Rev. 129 (1963) 2252.

[34] R. Nilson, R. O. Kerman, G. R. Briggs, and W. Jentschke, Phys. Rev. 104 (1956) 1673.

[35] F. E. Steigert and M. B. Sampson, Phys. Rev. 92 (1953) 660.

[36] W. S. Chien and R. E. Brown, Phys. Rev. C 10 (1974) 1767.

[37] R. M. DeVries and M. R. Clover, Nucl. Phys. A 243 (1975) 529; J. E. Poling, E. Norbeck, and R. R. Carlson, Phys. Rev. C 13 (1976) 648.

[38] M. Rhoades-Brown, M. H. Macfarlane, and S. C. Pieper, Phys. Rev. C 21 (1980) 2417; ibid. 2436.

[39] M. El-Azab Farid, Z. M. M. Mahmoud, and G. S. Hassan, Nucl. Phys. A 691 (2001) 671.

[40] M. Karakoc and I. Boztosun, Phys. Rev. C 73 (2006) 047601.

\section{APPENDIX: ANTISYMMETRIZED OVERLAP DENSITY OF THE $\alpha+\alpha$ SYSTEM}

We consider two $\alpha$ particles, whose centres of mass are separated by a distance $R$, in the Brink's microscopic cluster model for ${ }^{8} \mathrm{Be}$ [29]. The antisymmetrized total wave function of the $\alpha+\alpha$ system in this model is given explicitly as

$$
\Psi(R)=n_{0}(R) \mathcal{A}\left\{\psi_{\alpha_{1}} \times \psi_{\alpha_{2}}\right\}=\frac{n_{0}(R)}{\sqrt{4 ! 4 !}} \operatorname{det}\left\{\varphi_{1} \varphi_{2} \ldots \varphi_{8}\right\}
$$

where the single-nucleon state is determined as the $\mathrm{s}_{1 / 2}$ harmonic oscillator wave function

$$
\begin{aligned}
\varphi_{i} & =g\left(\boldsymbol{r}_{i}+\frac{\boldsymbol{R}}{2}, b\right) \chi_{i} \xi_{i} \quad \text { if } \quad i=1, \ldots, 4 \\
\varphi_{i} & =g\left(\boldsymbol{r}_{i}-\frac{\boldsymbol{R}}{2}, b\right) \chi_{i} \xi_{i} \quad \text { if } \quad i=5, \ldots, 8 \\
g(\boldsymbol{r}, b) & =\frac{1}{\pi^{3 / 4} b^{3 / 2}} \exp \left(-\frac{\boldsymbol{r}^{2}}{2 b^{2}}\right) .
\end{aligned}
$$

The h.o. parameter $b=1.1932 \mathrm{fm}[15], \mathcal{A}$ is the antisymmetrizing operator, $\chi_{i}$ and $\xi_{i}$ are the spin and isospin wave functions of the $i$-th nucleon, and $n_{0}(R)$ is the normalization constant determined from the condition

$$
\langle\Psi(R) \mid \Psi(R)\rangle=n_{0}(R)^{2} \frac{8 !}{4 ! 4 !}\left\langle\Psi_{8} \mid \mathcal{A} \Psi_{8}\right\rangle=n_{0}(R)^{2} \frac{8 !}{4 ! 4 !} \operatorname{det} B=1 .
$$

Here $\Psi_{8}=\varphi_{1} \ldots \varphi_{8}, \mathcal{A} \Psi_{8}=\operatorname{det}\left\{\varphi_{1} \ldots \varphi_{8}\right\}$, and the matrix elements of $B$ are

$$
\begin{aligned}
B_{i j}=\left\langle\varphi_{i} \mid \varphi_{j}\right\rangle & =1 & & \text { if } j=i, \\
& =\exp \left(-\frac{R^{2}}{4 b^{2}}\right) & & \text { if } j=i \pm 4, \\
& =0 & & \text { otherwise. }
\end{aligned}
$$


The antisymmetrized overlap density of the $\alpha+\alpha$ system is determined as

$$
\begin{aligned}
\rho(\boldsymbol{r}, R) & =\left\langle\Psi(R)\left|\sum_{i=1}^{8} \delta\left(\boldsymbol{r}-\boldsymbol{r}_{i}\right)\right| \Psi(R)\right\rangle \\
& =n_{0}(R)^{2} \frac{8 !}{4 ! 4 !}\left\langle\Psi_{8}\left|\sum_{i=1}^{8} \delta\left(\boldsymbol{r}-\boldsymbol{r}_{i}\right)\right| \mathcal{A} \Psi_{8}\right\rangle \\
& =\sum_{i=1}^{8} \sum_{j=1}^{8}\left\langle\varphi_{i}\left|\delta\left(\boldsymbol{r}-\boldsymbol{r}_{i}\right)\right| \varphi_{j}\right\rangle\left(B^{-1}\right)_{j i},
\end{aligned}
$$

where the matrix elements of the $\delta$-function are

$$
\begin{aligned}
\left\langle\varphi_{i}\left|\delta\left(\boldsymbol{r}-\boldsymbol{r}_{i}\right)\right| \varphi_{j}\right\rangle & =\frac{1}{\pi^{3 / 2} b^{3}} \exp \left[-\frac{1}{b^{2}}\left(\boldsymbol{r} \pm \frac{\boldsymbol{R}}{2}\right)^{2}\right] & \text { if } j=i \\
& =\frac{1}{\pi^{3 / 2} b^{3}} \exp \left[-\frac{1}{b^{2}}\left(r^{2}+\frac{R^{2}}{4}\right)\right] & \text { if } j=i \pm 4 \\
& =0 & \text { otherwise. }
\end{aligned}
$$

In the diagonal matrix elements, the sign is $(+)$ when $i=j \leqslant 4$ and $(-)$ when $i=j \geqslant 5$. Using the explicit expression for $\left(B^{-1}\right)_{j i}$ derived directly from Eq. (20), we obtain the $\alpha+\alpha$ overlap density (22) in the following compact form

$$
\begin{aligned}
\rho(\boldsymbol{r}, R) & =\frac{4}{\pi^{3 / 2} b^{3}[1-h(R, b)]}\left\{\exp \left[-\frac{1}{2 b^{2}}\left(\boldsymbol{r}-\frac{\boldsymbol{R}}{2}\right)^{2}\right]-2 \exp \left(-\frac{r^{2}}{b^{2}}\right) h(R, b)\right. \\
& \left.+\exp \left[-\frac{1}{2 b^{2}}\left(\boldsymbol{r}+\frac{\boldsymbol{R}}{2}\right)^{2}\right]\right\}, \text { with } h(R, b)=\exp \left(-\frac{R^{2}}{2 b^{2}}\right)
\end{aligned}
$$

Like the h.o model for the $\alpha$-density discussed in Sec. II, this model for the $\alpha+\alpha$ overlap density can also be corrected for the center-of-mass motion using the prescription of Ref. [18], and the h.o. range of the Gaussians in Eq. (18) will be modified accordingly. However, all the results would be the same if we keep using the same empirical h.o. parameter $b=1.1932 \mathrm{fm}$ in both cases without c.m. motion correction. 